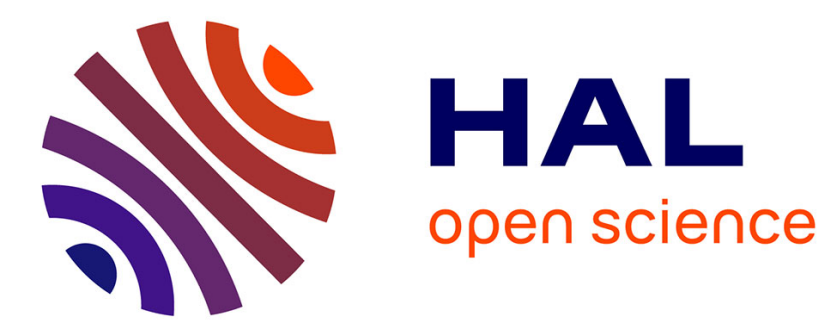

\title{
Radical 1,5-Chloropentafluorosulfanylation of Unactivated Vinylcyclopropanes and Transformation into $\alpha$-SF 5 Ketones
}

Fang-Fang Feng, Jun-An Ma, Dominique Cahard

\section{To cite this version:}

Fang-Fang Feng, Jun-An Ma, Dominique Cahard. Radical 1,5-Chloropentafluorosulfanylation of Unactivated Vinylcyclopropanes and Transformation into $\alpha$-SF 5 Ketones. Journal of Organic Chemistry, In press, 10.1021/acs.joc.1c01886 . hal-03357178

\section{HAL Id: hal-03357178 \\ https://hal.science/hal-03357178}

Submitted on 30 Sep 2021

HAL is a multi-disciplinary open access archive for the deposit and dissemination of scientific research documents, whether they are published or not. The documents may come from teaching and research institutions in France or abroad, or from public or private research centers.
L'archive ouverte pluridisciplinaire HAL, est destinée au dépôt et à la diffusion de documents scientifiques de niveau recherche, publiés ou non, émanant des établissements d'enseignement et de recherche français ou étrangers, des laboratoires publics ou privés. 


\title{
Radical 1,5-Chloropentafluorosulfanylation of Unactivated Vinylcyclopropanes and Transformation into $\alpha-\mathrm{SF}_{5}$ Ketones
}

\author{
Fang-Fang Feng, ${ }^{\dagger \dagger}$ Jun-An Ma, ${ }^{*},{\text { Dominique Cahard }{ }^{*}, \dagger}^{\dagger}$ \\ ${ }^{\dagger}$ UMR 6014 CNRS COBRA, Normandie Université, INSA Rouen, 1 rue Tesnière, 76821 Mont Saint Aignan, France \\ * Department of Chemistry, Tianjin Key Laboratory of Molecular Optoelectronic Sciences, and Collaborative Innovation \\ Center of Chemical Science and Engineering, Tianjin University, Tianjin 300072, China
}

Supporting Information Placeholder

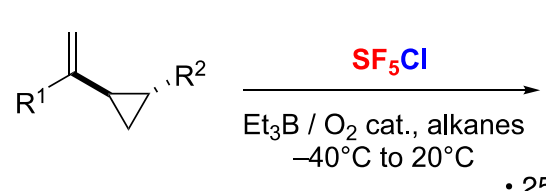

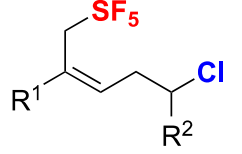

- 25 new compounds

- mild and simple conditions

- broad substrate scope and high yields

- regioselective addition \& ring-opening

ABSTRACT: The radical 1,5-chloropentafluorosulfanylation of vinyl cyclopropanes (VCPs) initiated by Et ${ }_{3} \mathrm{~B} / \mathrm{O}_{2}$ affords allylic pentafluorosulfanyl / homoallylic chloride products through ring-strain release of the cyclopropane. The VCP substitution pattern was investigated. The utility of this reaction was illustrated in post-transformation of the $\mathrm{C}=\mathrm{C}$ bond by ozonolysis giving access to valuable $\alpha-\mathrm{SF}_{5}$ carbonyl compounds.

Vinyl cyclopropane (VCP) is an all-carbon multifaceted scaffold of great interest for the installation of elements and a diversity of functional groups in straightforward synthetic transformations. ${ }^{1}$ The substitution pattern of VCPs strongly impacts the reactivity and reaction possibilities with nucleophilic, electrophilic or radical partners. Activated VCPs, which typically feature two geminal electron-withdrawing substituents (EWS) on the cyclopropane ring to ease ringopening reactions, react with the aid of transition-metal catalysts to form zwitterionic $\pi$-allylmetal intermediates that subsequently engage in allylic substitutions, addition reactions or cycloaddition reactions. ${ }^{2}$ In sharp contrast, unactivated VCPs lacking EWS less favourably form 1,3-dipoles in the presence of metal catalysts and prefer to undergo oxidative addition to form a metallacycle intermediate for subsequent annulation reactions. ${ }^{3}$ Unactivated VCPs, as five-carbon synthons, were explored in a number of transition-metal catalyzed 1,5difunctionalization reactions, e.g. Ni-catalyzed silaboration, ${ }^{4}$ Fe-catalyzed hydroboration, ${ }^{5}$ Fe-catalyzed dicarbofunctionalization, ${ }^{6} \mathrm{Rh}$-catalyzed nitrene-transfer, ${ }^{7} \mathrm{Cu}$ catalyzed cyanotrifluoromethylation, ${ }^{8} \quad$ Au-catalyzed hydroamination, ${ }^{9}$ and Ir-catalyzed bromotrichloromethylation. ${ }^{10}$ In addition, an organocatalytic $\left(\mathrm{C}_{6} \mathrm{~F}_{5}\right)_{3} \mathrm{~B}$-catalyzed ionic hydrosilylation ${ }^{11}$ and a visible-lightpromoted hydrotrifluoromethylation ${ }^{12}$ have been developed. In mechanistic studies, the VCP scaffold is widely used as radical probe but the 1,5-difunctionalized products are clearly not central to the research works. ${ }^{13}$ Remote 1,5 -difunctionalization of unactivated VCPs with concomitant creation of a $\mathrm{C}-\mathrm{S}$ bond has been reported in 1974 by reaction of arylsulfenyl chloride with VCPs through a carbonium ion intermediate ${ }^{14}$ and recently in 2020 in a photo-mediated organocatalytic radical 1,5trifluoromethylthio-sulfonylation. ${ }^{15}$ Other examples of $\mathrm{C}-\mathrm{S}$ bond formation starting from the VCP motif could be found in mechanistic studies of various reactions exploiting VCPs as radical probes. ${ }^{16}$ In order to increase the diversity of sulfurcontaining products accessible from VCPs, we herein report that exposure of unactivated VCPs to pentafluorosulfanyl chloride under metal-free conditions in the presence of a radical initiator resulted in an efficient, atom-economic 1,5chloropentafluorosulfanylation reaction (Scheme 1).

Scheme 1. Radical pentafluorosulfanylation of VCPs. ${ }^{a}$

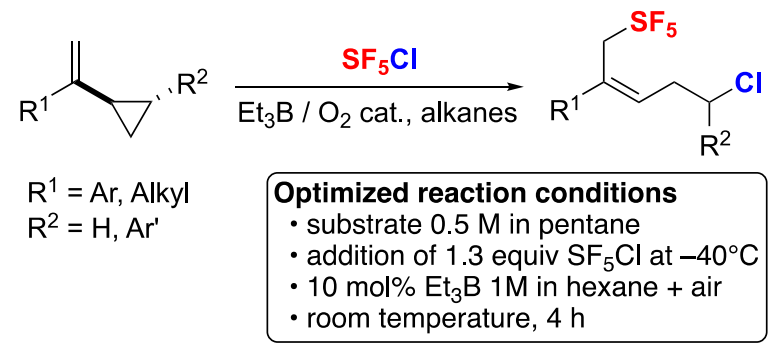

${ }^{a}$ See the experimental section for detailed procedures.

Our choice for the fluorine-containing $\mathrm{SF}_{5}$ motif was motivated by its emerging position in the ever more vibrant field of organofluorine chemistry. Indeed, the incremental progress of the $\mathrm{SF}_{5}$ chemistry has demonstrated that installation of a $\mathrm{SF}_{5}$ 
group on an organic molecule deeply influences its chemical and physical properties (reactivity, metabolic stability, membrane permeability, aqueous solubility, $\mathrm{pKa}, \log D, \ldots){ }^{17}$ Nevertheless, the number of $\mathrm{SF}_{5}$-containing molecules is still rather small and a particular focus is given to $\mathrm{SF}_{5}$-substituted (hetero)aromatics ${ }^{18}$ while aliphatic $\mathrm{SF}_{5}$ derivatives are less investigated. ${ }^{19}$ In this context, expanding the chemical space of $\mathrm{SF}_{5}$-containing aliphatic compounds offers wide opportunities. Specifically, we have used pentafluorosulfanyl chloride in a radical chain addition process to VCPs in the presence of the couple $\mathrm{Et}_{3} \mathrm{~B} / \mathrm{O}_{2}$ as a catalytic initiator.

$\mathrm{SF}_{5} \mathrm{Cl}$ is a colorless gas $\left(\mathrm{Bp}-21^{\circ} \mathrm{C}\right)$ commercially available from rare suppliers or prepared in the lab according to a recent procedure. $^{20}$ It can be easily condensed in hexane and the solutions are kept in a freezer for weeks. Since the demonstration by Dolbier and Aït-Mohand in 2002 that $\mathrm{Et}_{3} \mathrm{~B} / \mathrm{O}_{2}$ acts as an efficient $\mathrm{SF}_{5}$ radical initiator, the method has become prevalent in the free radical chain addition of $\mathrm{SF}_{5}{ }^{*}$ to unsaturations. $^{21 \mathrm{a}-\mathrm{c}}$ Note that the combination $\mathrm{Et}_{3} \mathrm{~B} / \mathrm{O}_{2}$ also served in the transfer of pyridine tetrafluorosulfanyl chloride to alkenes and alkynes, ${ }^{21 \mathrm{~d}}$ albeit it was later demonstrated that these precise reactions performed spontaneously without catalyst. $^{21 \mathrm{e}}$ It is therefore only natural that we carried out our study with the couple $\mathrm{Et}_{3} \mathrm{~B} / \mathrm{O}_{2}$ keeping in mind to evaluate the requirement of it. By selecting the unactivated VCPs $\mathbf{1}$ as substrates, which feature the vinyl group as radical accepting group and an aryl or an alkyl substituent as radical stabilizing group, we anticipated that one issue would be the participation or not of the cyclopropane ring to the chain process; in other words, either the 1,2-addition product 2 or the 1,5-addition product 3 could possibly formed (Scheme 2).

Scheme 2. Scope I of the pentafluorosulfanylation of VCPs: vinyl substituent effect. ${ }^{a, b}$

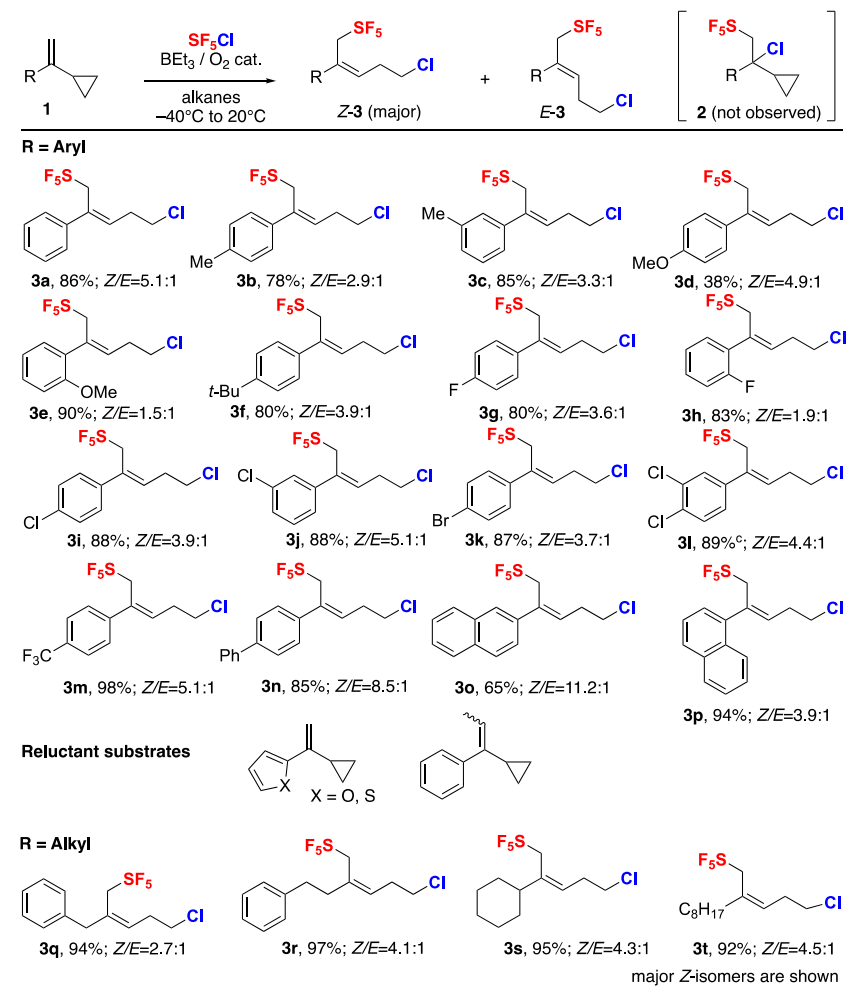

${ }^{\mathrm{a}}$ See experimental section for detailed procedures. ${ }^{\mathrm{b}} \mathrm{Z} / E$ ratios were determined on crude reaction mixtures. ${ }^{c}$ Two equivalents of $\mathrm{SF}_{5} \mathrm{Cl}$ were required to reach full conversion.

The experiments demonstrated the obtention of $\mathbf{3}$ as single regioisomer with $Z / E$ ratios depending on the substitution pattern of substrates $\mathbf{1}$. The $Z$-isomer was assigned as the major isomer by 2D NOESY NMR of product $\mathbf{3 n}$ and X-ray crystal analysis of product 3o. ${ }^{22}$ Consistently, high yields were achieved with the variously substituted phenyl groups in 3a-p with one exception (3d was obtained in $38 \%$ yield). Halogens (F, $\mathrm{Cl}, \mathrm{Br})$, alkyls $(\mathrm{Me}, t-\mathrm{Bu})$, aryl (Ph, Naphth), EDG and EWG groups $\left(\mathrm{MeO}, \mathrm{CF}_{3}\right)$ at different ring positions are compatible with the reaction conditions. Nevertheless, we identified reluctant substrates that are heteroaryl-containing VCPs as well as a VCP featuring a trisubstituted olefin moiety (Scheme 2). In the cases of 2-furyl- and 2-thiophenyl-derived VCPs, the substrates were fully consumed yet not in the expected allylic $\mathrm{SF}_{5}$ products. The reaction conditions were modified, in particular by using other solvents. In ethyl acetate, two major products were identified resulting in 1,5acetylchlorination and 1,5-chlorofluorination. The required acetyl radical was generated by homolytic cleavage of EtOAc and the fluorine radical was formed together with $\mathrm{SF}_{4}$ (detected by ${ }^{19} \mathrm{~F} \mathrm{NMR}$ ) during the decomposition of $\mathrm{SF}_{5}$ radical. Possibly, the Lewis acidity of triethylborane was an issue for these heteroaryl substrates. Alkyl-substituted VCPs gave even higher yields $(92-97 \%)$ in 1,5-chloropentafluorosulfanylated products 3q-t (Scheme 2). The explanation could be that the tertiary radical intermediates are less stabilized than the benzylic radical issued of aryl substituted VCPs and thus they more easily promote the radical-mediated cyclopropane ringopening reaction affording the homoallylic primary radical (see later in the text Scheme 4).

A further scope investigation was carried out with disubstituted trans-cyclopropyl VCPs. Here again the regioselective 1,5 -addition products were isolated as sole products; moreover, the cyclopropane ring-opening furnished specifically products $3 \mathbf{u}-\mathbf{x}$ without trace of isomers $\mathbf{4}$ as certified by COSY NMR (Scheme 3 and SI). As previously noticed with alkyl VCPs 3q-t, addition product $\mathbf{3 y}$ was obtained in an excellent $94 \%$ yield, and furthermore with a total diastereoselectivity for the $Z$ isomer.

Scheme 3. Scope II of the pentafluorosulfanylation of VCPs: cyclopropyl substituent effect. ${ }^{a, b}$

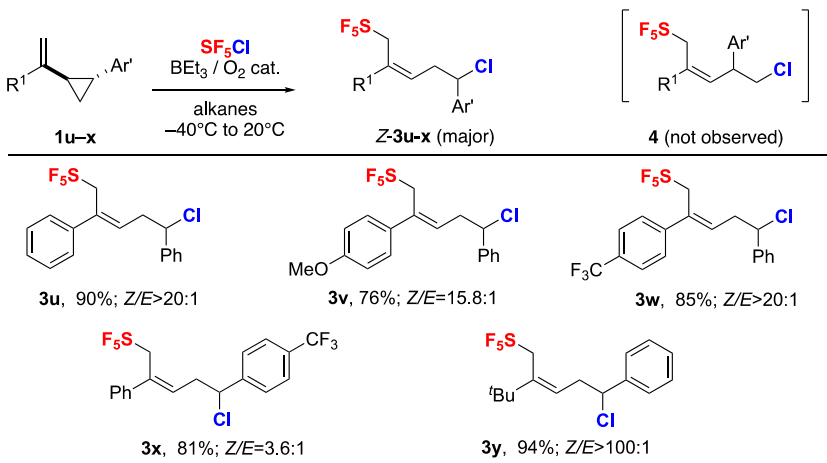

major $Z$-isomers are shown

${ }^{\mathrm{a}}$ See experimental section for detailed procedures. ${ }^{\mathrm{b}} Z / E$ ratios were determined on crude reaction mixtures. 
From a mechanistic point of view, triethylborane underwent a homolytic substitution $\left(\mathrm{S}_{\mathrm{H}} 2\right)$ reaction with triplet $\mathrm{O}_{2}$ from which an ethyl radical was liberated. When in contact with $\mathrm{SF}_{5} \mathrm{Cl}, \mathrm{Et}^{\circ}$

Scheme 4. Mechanistic suggestions.

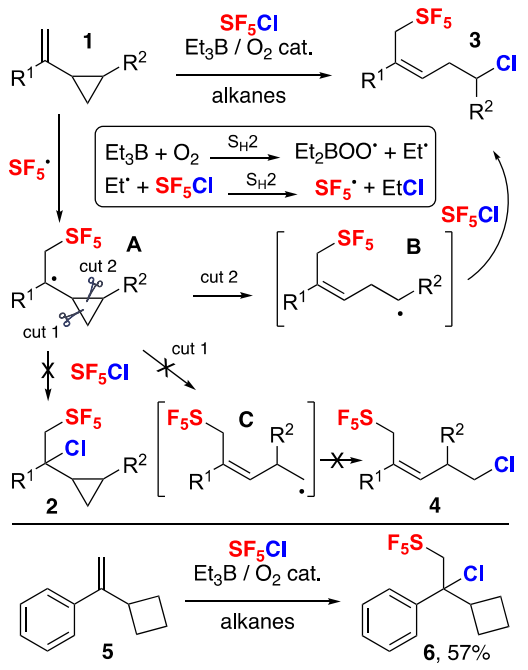

caused the $\mathrm{S}_{\mathrm{H}} 2$ to produce the radical $\mathrm{SF}_{5}{ }^{\cdot}$ and $\mathrm{EtCl}$ (Scheme 4). VCPs reacted regioselectively at the vinyl group with $\mathrm{SF}_{5}{ }^{*}$ to furnish the cyclopropylcarbinyl radical $\mathbf{A}$ and further ringopening by $\beta$-homolytic fission and strain release rearrangement to give $\mathbf{B}$, which propagates the chain process by $\mathrm{S}_{\mathrm{H}} 2$ with $\mathrm{SF}_{5} \mathrm{Cl}$ and production of compound 3 . The reverse reaction that releases the double bond was slower than the ringopening and $\beta$-fragmentation of the pentafluorothiyl radical was not a limitation. Since the release of strain from the cyclopropane ring is thermodynamically highly favorable, product 2 resulting in a 1,2-addition onto the vinyl function was not observed. For substrates featuring a $\mathrm{R}^{2}$ substituent on the cyclopropane moiety, two regioisomers could potentially be obtained: $\mathbf{3}$ via the secondary alkyl radical $\mathbf{B}$ and $\mathbf{4}$ via the primary alkyl radical $\mathbf{C}$. The greater stability of $\mathbf{B}$ is responsible for the unique formation of addition product $\mathbf{3}$ (Scheme 4). In the absence of $\mathrm{Et}_{3} \mathrm{~B} / \mathrm{O}_{2}$, no spontaneous reaction took place. Regarding the diastereoselectivity, the ratio $Z / E$ was dictated by the conformation of the intermediate cyclopropylcarbinyl radical $\mathbf{A}$ and varies with the VCP substitution pattern. ${ }^{23} \mathrm{Ap}$ plication of the chloropentafluorosulfanylation to homologous vinylcyclobutane was somehow successful; however, while both the vinyl and the cyclopropane participate in the 1,5difunctionalization of VCP, the cyclobutane ring in homologue vinylcyclobutane 5 was not altered and the 1,2-addition product $\mathbf{6}$ was isolated as single reaction product (Scheme 4).

The potential of $\alpha-\mathrm{SF}_{5}$ carbonyl compounds, for instance in enolate chemistry, has not yet been explored in details because of the paucity of synthetic methods to reach these structures. Pioneering works by Seppelt consisted of $\mathrm{SF}_{5} \mathrm{Cl}$ addition onto diketene and hydrolysis of the intermediate ${ }^{24 a}$ or on ketene followed by a Friedel-Crafts reaction (Scheme 5). ${ }^{24 \mathrm{~b}} \mathrm{The}_{\mathrm{Et}} \mathrm{B}$ catalyzed addition of $\mathrm{SF}_{5} \mathrm{Cl}$ onto enol acetates of acetone by Dolbier $^{24 c}$ or of acetophenone by Welch ${ }^{24 d}$ gave after hydroly- sis the corresponding $\alpha-\mathrm{SF}_{5}$ ketones. Among the most recent approaches toward $\alpha-\mathrm{SF}_{5}$ ketones, one can cite the goldcatalyzed hydration of $\mathrm{SF}_{5}$-alkynes described by Paquin ${ }^{24 \mathrm{e}}$ and the direct pentafluorosulfanylation of diazo compounds in the presence of tripotassium phosphate by Qing (Scheme 5). ${ }^{24 \mathrm{f}} \mathrm{We}$ assumed that allylic pentafluorosulfanyl products $\mathbf{3}$ would be precursors

Scheme 5. Preparation of $\alpha-\mathrm{SF}_{5}$ ketones: State-of-the-art and our approach by ozonolysis.

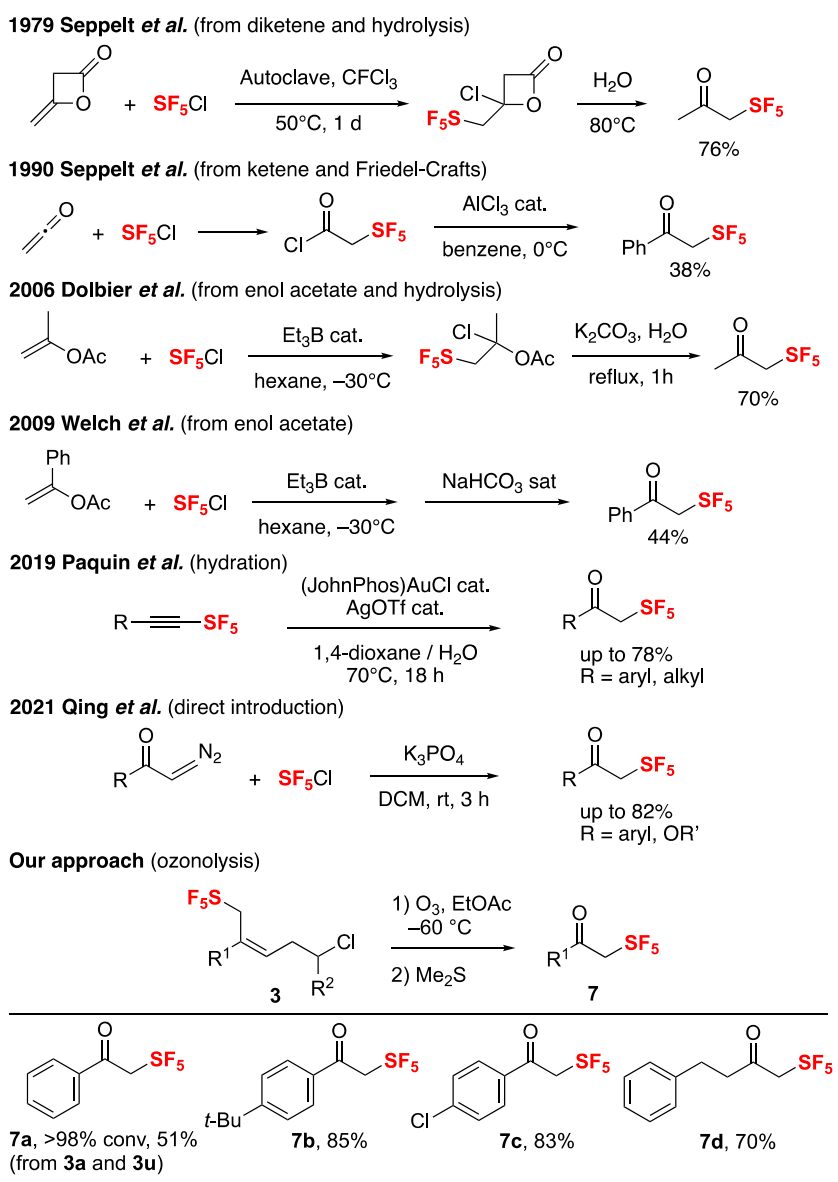

${ }^{a}$ See experimental section for detailed procedures.

of $\alpha-\mathrm{SF}_{5}$ ketones through ozonolysis. Indeed, aryl and aliphatic ketones 7a-d were easily obtained by ozonolysis in ethyl acetate with reductive workup (Scheme 5). The transformation was clean and quantitative from $\mathbf{3 a}$ and $\mathbf{3} \mathbf{u}$ affording the same ketone $7 \mathbf{a}$ albeit in a moderate yield due to its volatility. Other $\alpha-\mathrm{SF}_{5}$ ketones with higher molecular weights were isolated in $70-85 \%$ yields. Notably, this transformation is the first evidence of compatibility of aliphatic $\mathrm{SF}_{5}$ derivatives with ozone.

In conclusion, we have carried out the radical 1,5chloropentafluorosulfanylation of vinyl cyclopropanes by means of a stock solution of $\mathrm{SF}_{5} \mathrm{Cl}$ and the catalytic combination $\mathrm{Et}_{3} \mathrm{~B} / \mathrm{O}_{2}$. Novel allylic pentafluorosulfanyl derivatives were prepared in high yields. Because the activation step is metal-free, catalytic in radical initiator and the 1,5-addition is obviously fully atom-economical, this process fulfills the key ideals of modern synthetic chemistry. Remarkably, a new 
synthetic strategy for the construction of $\alpha-\mathrm{SF}_{5}$ ketones has been established. The key elements of this cutting-edge research in organofluorine compounds contributes to the flourishing chemistry of the emerging $\mathrm{SF}_{5}$ motif.

\section{EXPERIMENTAL SECTION}

All experiments were conducted under a fume-hood. Analytical thin-layer chromatographies were performed on silica gel aluminum plates with F-254 indicator and visualized by UV light $(254 \mathrm{~nm})$ and/or chemical staining with a $p$-anisaldehyde or $\mathrm{KMnO}_{4}$ solution. Column chromatographies were performed with silica gel $(0.040-0.060 \mathrm{~nm})$. NMR spectra were recorded on a Brucker DXP 300 instrument at $300 \mathrm{MHz}$ for ${ }^{1} \mathrm{H}, 75.5 \mathrm{MHz}$ for ${ }^{13} \mathrm{C}$ and $282 \mathrm{MHz}$ for ${ }^{19} \mathrm{~F}$ in $\mathrm{CDCl}_{3}$ at room temperature unless otherwise stated. Chemical shifts $(\delta)$ were quoted in parts per million (ppm) relative to the residual peak of $\mathrm{CHCl}_{3}\left(\delta_{\mathrm{H}}=7.26 \mathrm{ppm}\right.$ and $\left.\delta_{\mathrm{C}}=77.16 \mathrm{ppm}\right)$ and internal standard $\mathrm{CFCl}_{3}\left(\delta_{\mathrm{F}}=0.00 \mathrm{ppm}\right)$. Spectra are reported as follows: chemical shift $\delta$ (ppm), multiplicity ( $\mathrm{s}=$ singlet, $\mathrm{d}=$ doublet, $\mathrm{dd}=$ doublet of doublets, $\mathrm{t}=$ triplet, $\mathrm{td}=$ triplet of doublets $\mathrm{q}=$ quartet, $\mathrm{p}=$ pentet, $\mathrm{m}=$ multiplet and $\mathrm{dm}=$ doublet of multiplet), integration and coupling constant. Infrared spectra were recorded on a PerkinElmer Spectrum 100. Highresolution mass spectra (HRMS) were recorded on a JEOL AccuTof 4G with GC HP Agilent 7890. Commercially available chemicals were obtained from Fisher scientific, Sigma Aldrich, Alfa Aesar, TCI, Fluorochem and used as received unless otherwise stated. Tetrahydrofuran (THF), diethyl ether $\left(\mathrm{Et}_{2} \mathrm{O}\right)$, and toluene were distilled from sodium / benzophenone prior to use; $\mathrm{CH}_{2} \mathrm{Cl}_{2}$ was distilled from $\mathrm{CaH}_{2}$.

\section{Synthesis of vinylcyclopropanes}

Vinylcyclopropanes 1a-p were prepared in a two-step sequence according to reported procedures. ${ }^{25 a, b}$ Vinylcyclopropanes 1q-t were prepared in a two-step sequence according to a reported procedure. ${ }^{26}$ Vinylcyclopropanes 1u-y were prepared in a two-step sequence according to a reported procedure. ${ }^{27}$ All vinylcyclopropanes $\mathbf{1 a - k}$ and $\mathbf{1 m - t}, \mathbf{1 u}, \mathbf{1 v}, \mathbf{1 x}$ and $\mathbf{1 y}$ are known compounds for which analytical data are identical to those reported in the literature. 1a CAS [825-76-3], ${ }^{25 b}$ 1b CAS [15620-28-7], ${ }^{25 b}$ 1c CAS [2374777-09-8], ${ }^{25 b}$ 1d CAS [829-174], ${ }^{25 b} \mathbf{1 e}$ CAS [1355988-71-4], ${ }^{25 b} \mathbf{1 f}$ CAS [933054-41-2], ${ }^{25 b} \mathbf{1 g}$ CAS [827-87-2], ${ }^{25 b}$ 1h CAS [2414140-70-6], ${ }^{25 b}$ 1i CAS [100933-2], ${ }^{25 b} \mathbf{1 j}$ CAS [2417453-22-4], ${ }^{25 b}$ 1k CAS [65322-32-9], ${ }^{25 b}$ $1 m$ CAS [1028387-07-6], ${ }^{25 b}$ 1n CAS [949090-83-9], ${ }^{25 b}$ 1o CAS [155337-96-5], ${ }^{25 b}$ 1p CAS [418763-99-2], ${ }^{25 b}$ 1q CAS $[25522-55-8],{ }^{26}$ 1r CAS [473441-75-7], ${ }^{26}$ 1s CAS [102838717-8], ${ }^{26}$ 1t CAS [1185742-70-4], ${ }^{26} \mathbf{1 u}$ CAS [50990-42-6], ${ }^{27} \mathbf{1 v}$ CAS [2135721-72-9], ${ }^{27}$ 1x CAS [2304809-66-1], ${ }^{27}$ 1y CAS [2381228-04-0]. ${ }^{27}$ Vinylcyclopropanes $\mathbf{1 l}$ and $\mathbf{1 w}$ are new compounds.

1,2-Dichloro-4-(1-cyclopropylvinyl)benzene (1l): Colorless oil after column chromatography with petroleum ether / ethyl acetate $30: 1 \quad\left(\mathrm{R}_{\mathrm{f}}=0.5\right), 75 \%$ yield. ${ }^{1} \mathbf{H}$ NMR $(300 \mathrm{MHz}$, $\left.\mathrm{CDCl}_{3}\right) \delta 7.68(\mathrm{dd}, J=1.9,0.6 \mathrm{~Hz}, 1 \mathrm{H}), 7.54-7.31(\mathrm{~m}, 2 \mathrm{H})$, $5.30(\mathrm{~d}, J=0.6 \mathrm{~Hz}, 1 \mathrm{H}), 5.00(\mathrm{dd}, J=1.3,0.7 \mathrm{~Hz}, 1 \mathrm{H}), 1.74-$ $1.43(\mathrm{~m}, 1 \mathrm{H}), 0.97-0.74(\mathrm{~m}, 2 \mathrm{H}), 0.68-0.43(\mathrm{~m}, 2 \mathrm{H})$. ${ }^{13} \mathbf{C}\left\{{ }^{1} \mathbf{H}\right\}$ NMR $\left(75 \mathrm{MHz}, \mathrm{CDCl}_{3}\right) \delta 147.3,141.7,132.3,131.4$, 130.1, 128.1, 125.5, 110.8, 15.5, 6.8. HRMS (EI) m/z: $\mathbf{M}^{+}$ Calcd for $\mathrm{C}_{11} \mathrm{H}_{11} \mathrm{Cl}_{2}$ 212.0160; Found 212.0158.

trans-1-(1-(2-Phenylcyclopropyl)vinyl)-4-

(trifluoromethyl)benzene (1w): Colorless oil after column chromatography with petroleum ether / ethyl acetate $30: 1\left(\mathrm{R}_{\mathrm{f}}\right.$ $=0.4), 80 \%$ yield. ${ }^{1} \mathbf{H}$ NMR $\left(300 \mathrm{MHz}, \mathrm{CDCl}_{3}\right) \delta 7.68(\mathrm{q}, J=$ $8.4 \mathrm{~Hz}, 4 \mathrm{H}), 7.42(\mathrm{t}, J=7.4 \mathrm{~Hz}, 2 \mathrm{H}), 7.32(\mathrm{~d}, J=7.0 \mathrm{~Hz}, 1 \mathrm{H})$, $7.28-7.19(\mathrm{~m}, 2 \mathrm{H}), 5.55(\mathrm{~s}, 1 \mathrm{H}), 5.26(\mathrm{~s}, 1 \mathrm{H}), 2.21-1.87(\mathrm{~m}$, $2 \mathrm{H}), 1.52(\mathrm{dt}, J=8.8,5.6 \mathrm{~Hz}, 1 \mathrm{H}), 1.42(\mathrm{dt}, J=8.7,5.5 \mathrm{~Hz}$, 1H). ${ }^{19} \mathbf{F}$ NMR $\left(282 \mathrm{MHz}, \mathrm{CDCl}_{3}\right) \delta-63.0 .{ }^{13} \mathbf{C}\left\{{ }^{1} \mathbf{H}\right\}$ NMR $(75$ $\left.\mathrm{MHz}, \mathrm{CDCl}_{3}\right) \delta 147.4,144.7(\mathrm{~d}, J=1.5 \mathrm{~Hz}), 142.3,129.9(\mathrm{t}, J$ $=32.4 \mathrm{~Hz}), 128.7,126.5,126.1,125.8,125.4(\mathrm{q}, J=3.8 \mathrm{~Hz})$, $124.4(\mathrm{~d}, J=271.8 \mathrm{~Hz}), 111.6,27.9,26.6,15.9$. HRMS (EI) $\mathrm{m} / \mathrm{z}: \mathrm{M}^{+\bullet}$ Calcd for $\mathrm{C}_{18} \mathrm{H}_{15} \mathrm{~F}_{3} 288.1126$; Found 288.1129.

\section{General procedure for the reaction of vinylcyclopropanes} 1 with $\mathrm{SF}_{5} \mathrm{Cl}$

To a vial $(10 \mathrm{~mL})$ equipped with a stirring bar was added vinylcyclopropane $1(0.5 \mathrm{mmol})$, and pentane $(1.0 \mathrm{~mL})$. The vial was then sealed. The mixture was stirred at $-40^{\circ} \mathrm{C}$, and a solution of $\mathrm{SF}_{5} \mathrm{Cl}$ in hexane $0.5-0.7 \mathrm{M}(0.76 \mathrm{mmol}, 1.34$ equiv) was added. Air was added into the system with a syringe. Then, $\mathrm{Et}_{3} \mathrm{~B} 1 \mathrm{M}$ in hexane ( $0.05 \mathrm{mmol}, 0.1$ equiv) was added dropwise. The reaction mixture was stirred at room temperature for $4 \mathrm{~h}$. Upon completion of the reaction (monitoring by TLC), the mixture was concentrated under vacuum. The residue was purified by flash chromatography on silica gel (eluting with petroleum ether/ethyl acetate) to give the desired products 3 , which are all new compounds.

(Z)-(5-chloro-2-phenylpent-2-en-1-yl)pentafluoro- $\lambda^{6}$-sulfane (Z-3a) and (E)-(5-chloro-2-phenylpent-2-en-1-yl)pentafluoro$\lambda^{6}$-sulfane (E-3a): Yellow liquid after column chromatography with petroleum ether / ethyl acetate 20:1 $\left(\mathrm{R}_{\mathrm{f}}=0.3\right)$. Yield ( $131.9 \mathrm{mg}, 0.43 \mathrm{mmol}, 86 \%$, mixture of $\mathrm{E} / \mathrm{Z}$ isomers $5.1: 1$ for the $0.5 \mathrm{mmol}, 72.04 \mathrm{mg}$ scale) and (1.306 g, $42.6 \mathrm{mmol}$, $85.4 \%$, mixture of $\mathrm{E} / \mathrm{Z}$ isomers $5.2: 1$ for the $50 \mathrm{mmol}, 720.47$ mg scale). ${ }^{1} \mathbf{H}$ NMR $\left(300 \mathrm{MHz}, \mathrm{CDCl}_{3}\right) \delta 7.37-7.16(\mathrm{~m}$, $4.54 \mathrm{H}), 7.16-7.05(\mathrm{~m}, 0.36 \mathrm{H}), 5.99(\mathrm{t}, \mathrm{J}=7.3 \mathrm{~Hz}, 0.76 \mathrm{H})$, $5.91(\mathrm{t}, \mathrm{J}=7.1 \mathrm{~Hz}, 0.17 \mathrm{H}), 4.67(\mathrm{p}, \mathrm{J}=7.6 \mathrm{~Hz}, 1.61 \mathrm{H}), 4.49$ $(\mathrm{p}, \mathrm{J}=7.7 \mathrm{~Hz}, 0.36 \mathrm{H}), 3.55(\mathrm{t}, \mathrm{J}=6.5 \mathrm{~Hz}, 1.61 \mathrm{H}), 3.40(\mathrm{t}, \mathrm{J}=$ $6.6 \mathrm{~Hz}, 0.34 \mathrm{H}), 2.68\left(\mathrm{q}_{\text {appt }}, \mathrm{J}=6.8 \mathrm{~Hz}, 1.62 \mathrm{H}\right), 2.45\left(\mathrm{q}_{\text {appt }}, \mathrm{J}=\right.$ $6.7 \mathrm{~Hz}, 0.37 \mathrm{H}) .{ }^{19} \mathbf{F}$ NMR $\left(282 \mathrm{MHz}, \mathrm{CDCl}_{3}\right) \delta 84.3-81.2$ $(\mathrm{m}, 1 \mathrm{~F}), 66.4(\mathrm{dm}, \mathrm{J}=145.0,3.35 \mathrm{~F}), 64.5(\mathrm{dm}, \mathrm{J}=146.6$, 0.65F). ${ }^{13} \mathbf{C}\left\{{ }^{1} \mathbf{H}\right\}$ NMR $\left(75 \mathrm{MHz}, \mathrm{CDCl}_{3}\right) \delta 140.5(\mathrm{Z}), 137.4$ (E), $135.7(\mathrm{E}), 135.2(\mathrm{Z}), 134.4(\mathrm{E})(\mathrm{p}, \mathrm{J}=2.2 \mathrm{~Hz}), 133.52(\mathrm{Z})$ $(\mathrm{p}, \mathrm{J}=2.0 \mathrm{~Hz}), 128.7(\mathrm{Z}), 128.6(\mathrm{E}), 128.5(\mathrm{E}), 128.1(\mathrm{Z})$, 128.0 (E), 126.6 (Z), 79.3 (E) (p, J = 12.4 Hz), 70.48 (Z) (p, J $=13.1 \mathrm{~Hz}), 43.5(\mathrm{E}), 43.3(\mathrm{Z}), 32.3(\mathrm{Z}+\mathrm{E})$. IR (ATR): $v_{\max }=$ 3030, 2964, 1692, 1599, 1495, 1447, 1298, 811, 775, 695, 645, 593, 563, $546 \mathrm{~cm}^{-1}$. HRMS (EI) m/z: $\mathrm{M}^{+\bullet}$ Calcd for $\mathrm{C}_{11} \mathrm{H}_{12} \mathrm{ClF}_{5} \mathrm{~S}$ 306.0268; Found 306.0284.

(Z)-(5-chloro-2-(p-tolyl)pent-2-en-1-yl)pentafluoro- $\lambda^{6}$-sulfane (Z-3b) and (E)-(5-chloro-2-phenylpent-2-en-1-yl)pentafluoro$\lambda^{6}$-sulfane (E-3b): Yellow liquid after column chromatography with petroleum ether / ethyl acetate 20:1 $\left(\mathrm{R}_{\mathrm{f}}=0.3\right)$, $125.1 \mathrm{mg}(0.39 \mathrm{mmol}), 78 \%$ yield, mixture of $\mathrm{E} / \mathrm{Z}$ isomers (2.9:1). ${ }^{1} \mathbf{H}$ NMR $\left(300 \mathrm{MHz}, \mathrm{CDCl}_{3}\right) \delta 7.20-7.10(\mathrm{~m}, 1.59 \mathrm{H})$, $7.14-7.03(\mathrm{~m}, 2 \mathrm{H}), 6.99(\mathrm{~d}, \mathrm{~J}=8.1 \mathrm{~Hz}, 0.57 \mathrm{H}), 5.97(\mathrm{t}, \mathrm{J}=$ $7.4 \mathrm{~Hz}, 0.74 \mathrm{H}), 5.89(\mathrm{t}, \mathrm{J}=7.1 \mathrm{~Hz}, 0.26 \mathrm{H}), 4.66(\mathrm{p}, \mathrm{J}=7.6 \mathrm{~Hz}$, $1.49 \mathrm{H}), 4.48(\mathrm{p}, \mathrm{J}=7.7 \mathrm{~Hz}, 0.54 \mathrm{H}), 3.55(\mathrm{t}, \mathrm{J}=6.5 \mathrm{~Hz}$, $1.47 \mathrm{H}), 3.40(\mathrm{t}, \mathrm{J}=6.6 \mathrm{~Hz}, 0.53 \mathrm{H}), 2.67\left(\mathrm{q}_{\mathrm{appt}}, \mathrm{J}=6.8 \mathrm{~Hz}\right.$, $1.51 \mathrm{H}), 2.46\left(\mathrm{q}_{\text {appt }}, \mathrm{J}=6.8 \mathrm{~Hz}, 0.60 \mathrm{H}\right), 2.26(\mathrm{~s}, 3 \mathrm{H}) .{ }^{19} \mathbf{F}$ NMR $\left(282 \mathrm{MHz}, \mathrm{CDCl}_{3}\right) \delta 84.1-80.2(\mathrm{~m}, 1 \mathrm{~F}), 66.4(\mathrm{dm}, \mathrm{J}=143.0$ $\mathrm{Hz}, 3.01 \mathrm{~F}), 64.4(\mathrm{dm}, \mathrm{J}=143.3 \mathrm{~Hz}, 1.03 \mathrm{~F}) .{ }^{13} \mathbf{C}\left\{{ }^{1} \mathbf{H}\right\} \mathbf{N M R}(75$ $\left.\mathrm{MHz}, \mathrm{CDCl}_{3}\right) \delta 138.0,137.8,137.7,135.4,134.5,134.4$, $134.4(\mathrm{p}, \mathrm{J}=2.2 \mathrm{~Hz}), 133.4(\mathrm{p}, \mathrm{J}=2.6 \mathrm{~Hz}), 129.4,129.3$, 128.3, 126.4, 79.4 (p, J = 12.2 Hz), 70.5 (p, J = 13.1 Hz), 43.6, 
43.3, 32.4, 32.4, 21.3, 21.1. HRMS (API $\left.{ }^{+}\right) \mathrm{m} / \mathrm{z}: \mathrm{M}^{+\bullet}$ Calcd for $\mathrm{C}_{12} \mathrm{H}_{14} \mathrm{ClF}_{5} \mathrm{~S} 320.0425$; Found 320.0420.

(Z)-(5-chloro-2-(m-tolyl)pent-2-en-1-yl)pentafluoro- $\lambda^{6}$ -

sulfane (Z-3c) and (E)-(5-chloro-2-(m-tolyl)pent-2-en-1yl)pentafluoro- $\lambda^{6}$-sulfane (E-3c): Yellow liquid after column chromatography with petroleum ether / ethyl acetate $20: 1\left(\mathrm{R}_{\mathrm{f}}\right.$ $=0.3), 137.0 \mathrm{mg}(0.43 \mathrm{mmol}), 85 \%$ yield, mixture of $\mathrm{E} / \mathrm{Z}$ isomers 3.3:1. ${ }^{1} \mathbf{H}$ NMR $\left(300 \mathrm{MHz}, \mathrm{CDCl}_{3}\right) \delta 7.23-7.09(\mathrm{~m}$, $1 \mathrm{H}), 7.10-6.99(\mathrm{~m}, 2.48 \mathrm{H}), 6.97-6.82(\mathrm{~m}, 0.53 \mathrm{H}), 5.98(\mathrm{t}, \mathrm{J}$ $=7.4 \mathrm{~Hz}, 0.74 \mathrm{H}), 5.90(\mathrm{t}, \mathrm{J}=7.1 \mathrm{~Hz}, 0.25 \mathrm{H}), 4.66(\mathrm{p}, \mathrm{J}=7.6$ $\mathrm{Hz}, 1.53 \mathrm{H}), 4.49(\mathrm{p}, \mathrm{J}=7.7 \mathrm{~Hz}, 0.52 \mathrm{H}), 3.55(\mathrm{t}, \mathrm{J}=6.5 \mathrm{~Hz}$, $1.51 \mathrm{H}), 3.40(\mathrm{t}, \mathrm{J}=6.6 \mathrm{~Hz}, 0.49 \mathrm{H}), 2.68(\mathrm{q}, \mathrm{J}=6.8 \mathrm{~Hz}$, $1.54 \mathrm{H}), 2.46(\mathrm{q}, \mathrm{J}=6.8 \mathrm{~Hz}, 0.54 \mathrm{H}), 2.27(\mathrm{~s}, 3 \mathrm{H}) .{ }^{19} \mathbf{F}$ NMR $\left(282 \mathrm{MHz}, \mathrm{CDCl}_{3}\right) \delta 83.8-81.0(\mathrm{~m}, 1 \mathrm{~F}), 66.3(\mathrm{dm}, \mathrm{J}=145.1$ $\mathrm{Hz}, 3.08 \mathrm{~F}), 64.5$ (dm, J = $144.8 \mathrm{~Hz}, 0.93 \mathrm{~F}) .{ }^{13} \mathbf{C}\left\{{ }^{1} \mathbf{H}\right\}$ NMR $(75$ $\left.\mathrm{MHz}, \mathrm{CDCl}_{3}\right) \delta 140.5,138.4,138.2,137.4,135.5,134.9$, $134.5(\mathrm{p}, \mathrm{J}=2.4 \mathrm{~Hz}), 133.7(\mathrm{p}, \mathrm{J}=2.6 \mathrm{~Hz}), 129.0,128.9$, 128.8, 128.6, 128.4, 127.4, 125.6, 123.6, 79.3 (p, J = 12.0 Hz), $70.5\left(\mathrm{p}, \mathrm{J}=13.3 \mathrm{~Hz}\right.$ ). HRMS (EI) $\mathrm{m} / \mathrm{z}: \mathrm{M}^{+\bullet}$ Calcd for $\mathrm{C}_{12} \mathrm{H}_{14} \mathrm{ClF}_{5} \mathrm{~S} 320.0425$; Found 320.0419.

(Z)-(5-chloro-2-(4-methoxyphenyl)pent-2-en-1-

yl)pentafluoro- $\lambda^{6}$-sulfane (Z-3d) and (E)-(5-chloro-2-(4methoxyphenyl)pent-2-en-1-yl)pentafluoro- $\lambda^{6}$-sulfane (E-3d): Yellow solid after column chromatography with petroleum ether / ethyl acetate $20: 1\left(\mathrm{R}_{\mathrm{f}}=0.2\right), 64.0 \mathrm{mg}(0.19 \mathrm{mmol})$, $38 \%$ yield, mixture of E/Z isomers 4.9:1. ${ }^{1} \mathbf{H}$ NMR $(300 \mathrm{MHz}$, $\left.\mathrm{CDCl}_{3}\right) \delta 7.35-7.24(\mathrm{~m}, 1.61 \mathrm{H}), 7.18-7.08(\mathrm{~m}, 0.39 \mathrm{H}), 7.00$ $-6.84(\mathrm{~m}, 2.07 \mathrm{H}), 6.03(\mathrm{t}, \mathrm{J}=7.4 \mathrm{~Hz}, 0.85 \mathrm{H}), 5.97(\mathrm{t}, \mathrm{J}=7.1$ $\mathrm{Hz}, 0.15 \mathrm{H}), 4.75(\mathrm{p}, \mathrm{J}=7.6 \mathrm{~Hz}, 1.67 \mathrm{H}), 4.76(\mathrm{p}, \mathrm{J}=7.5 \mathrm{~Hz}$, $0.47 \mathrm{H}), 3.83(\mathrm{~s}, 0.66 \mathrm{H}), 3.82(\mathrm{~s}, 2.23 \mathrm{H}), 3.67(\mathrm{t}, \mathrm{J}=6.5 \mathrm{~Hz}$, $1.56 \mathrm{H}), 3.52(\mathrm{t}, \mathrm{J}=6.6 \mathrm{~Hz}, 0.38 \mathrm{H}), 2.78(\mathrm{q}, \mathrm{J}=6.8 \mathrm{~Hz}$, $1.62 \mathrm{H}), 2.58(\mathrm{q}, \mathrm{J}=6.8 \mathrm{~Hz}, 0.39 \mathrm{H}) .{ }^{19} \mathbf{F} \mathbf{N M R}(282 \mathrm{MHz}$, $\left.\mathrm{CDCl}_{3}\right) \delta 85.8-79.1(\mathrm{~m}, 1 \mathrm{~F}), 66.4(\mathrm{dm}, \mathrm{J}=143.0 \mathrm{~Hz}, 3.30 \mathrm{~F})$, $64.4(\mathrm{dm}, \mathrm{J}=146.5 \mathrm{~Hz}, 0.68 \mathrm{~F}) .{ }^{13} \mathbf{C}\left\{{ }^{1} \mathbf{H}\right\}$ NMR $(75 \mathrm{MHz}$, $\left.\mathrm{CDCl}_{3}\right) \delta 159.5,159.2,135.2(\mathrm{p}, \mathrm{J}=2.2 \mathrm{~Hz}), 133.6,132.9(\mathrm{p}, \mathrm{J}$ $=2.5 \mathrm{~Hz}), 129.6,127.6,114.0,113.9,79.2(\mathrm{q}, \mathrm{J}=12.3 \mathrm{~Hz})$, $70.5(\mathrm{q}, \mathrm{J}=13.2 \mathrm{~Hz}), 55.3,55.3,43.5,43.3,32.4,32.3$. HRMS (EI) $\mathrm{m} / \mathrm{z}$ : $\mathrm{M}^{+}$Calcd for $\mathrm{C}_{12} \mathrm{H}_{14} \mathrm{ClF}_{5} \mathrm{OS} 336.0374$; Found 336.0376.

(Z)-(5-chloro-2-(2-methoxyphenyl)pent-2-en-1-

yl)pentafluoro- $\lambda^{6}$-sulfane (Z-3e) and (E)-(5-chloro-2-(2methoxyphenyl)pent-2-en-1-yl)pentafluoro- $\lambda^{6}$-sulfane (E-3e): Yellow oil after column chromatography with petroleum ether / ethyl acetate $20: 1\left(\mathrm{R}_{\mathrm{f}}=0.2\right), 151.5 \mathrm{mg}(0.45 \mathrm{mmol}), 90 \%$ yield, mixture of E/Z isomers 1.5:1. ${ }^{1} \mathbf{H}$ NMR $(300 \mathrm{MHz}$, $\left.\mathrm{CDCl}_{3}\right) \delta 7.34$ (dddd, $\left.\mathrm{J}=8.2,7.4,4.6,1.8 \mathrm{~Hz}, 1 \mathrm{H}\right), 7.25-7.10$ $(\mathrm{m}, 1 \mathrm{H}), 7.07-6.86(\mathrm{~m}, 2 \mathrm{H}), 6.05(\mathrm{t}, \mathrm{J}=7.2 \mathrm{~Hz}, 0.41 \mathrm{H}), 5.94$ (t, J = 7.3 Hz, 0.59H), $4.93(\mathrm{p}, \mathrm{J}=8.0 \mathrm{~Hz}, 1.24 \mathrm{H}), 4.71(\mathrm{p}, \mathrm{J}=$ $8.1 \mathrm{~Hz}, 0.77 \mathrm{H}), 3.84(\mathrm{~s}, 1.76 \mathrm{H}), 3.83(\mathrm{~s}, 1.19 \mathrm{H}), 3.67(\mathrm{t}, \mathrm{J}=$ $6.7 \mathrm{~Hz}, 1.20 \mathrm{H}), 3.52(\mathrm{t}, \mathrm{J}=6.7 \mathrm{~Hz}, 0.81 \mathrm{H}), 2.80(\mathrm{q}, \mathrm{J}=6.9 \mathrm{~Hz}$, $1.20 \mathrm{H}), 2.51(\mathrm{q}, \mathrm{J}=6.9 \mathrm{~Hz}, 0.8 \mathrm{H}) .{ }^{19} \mathbf{F} \mathbf{N M R}(282 \mathrm{MHz}$, $\left.\mathrm{CDCl}_{3}\right) \delta 86.4-80.6(\mathrm{~m}, 1 \mathrm{~F}), 66.9(\mathrm{dm}, \mathrm{J}=143.1,2.46 \mathrm{~F})$, $64.5(\mathrm{dm}, \mathrm{J}=144.4,1.58 \mathrm{~F}) .{ }^{13} \mathbf{C}\left\{{ }^{1} \mathbf{H}\right\}$ NMR $\left(75 \mathrm{MHz}, \mathrm{CDCl}_{3}\right)$ $\delta 156.6,156.4,136.4,132.9(\mathrm{p}, \mathrm{J}=2.7 \mathrm{~Hz}), 132.2(\mathrm{p}, \mathrm{J}=2.6$ Hz), 131.4, 131.3, 130.0, 129.6, 129.5, 125.5, 121.1, 120.6, 111.0, 110.6, 78.1 (p, J = 11.6 Hz), 70.8 (p, J = 12.5 Hz), 55.4, 43.5, 43.2, 32.5, 32.0. HRMS (EI) $\mathrm{m} / \mathrm{z}: \mathrm{M}^{+\bullet}$ Calcd for $\mathrm{C}_{12} \mathrm{H}_{14} \mathrm{ClF}_{5} \mathrm{OS} 336.0374$; Found 336.0377.

(Z)-(2-(4-(tert-butyl)phenyl)-5-chloropent-2-en-1-

yl)pentafluoro- $\lambda^{6}$-sulfane (Z-3f) and (E)-(2-(4-(tertbutyl)phenyl)-5-chloropent-2-en-1-yl)pentafluoro- $\lambda^{6}$-sulfane (E-3f): Yellow oil after column chromatography with petrole- um ether / ethyl acetate 20:1 $\left(\mathrm{R}_{\mathrm{f}}=0.3\right), 156.0 \mathrm{mg}(0.43$ mmol), $86 \%$ yield, mixture of $E / Z$ isomers $3.9: 1 .{ }^{1} \mathbf{H}$ NMR $\left(300 \mathrm{MHz}, \mathrm{CDCl}_{3}\right) \delta 7.49-7.38(\mathrm{~m}, 2 \mathrm{H}), 7.39-7.29(\mathrm{~m}$, $1.70 \mathrm{H}), 7.22-7.12(\mathrm{~m}, 0.41 \mathrm{H}), 6.13(\mathrm{t}, \mathrm{J}=7.4 \mathrm{~Hz}, 0.81 \mathrm{H})$, $6.04(\mathrm{t}, \mathrm{J}=7.1 \mathrm{~Hz}, 0.19 \mathrm{H}), 4.81(\mathrm{p}, \mathrm{J}=7.6 \mathrm{~Hz}, 1.61 \mathrm{H}), 4.63$ $(\mathrm{p}, \mathrm{J}=7.7 \mathrm{~Hz}, 0.41 \mathrm{H}), 3.68(\mathrm{t}, \mathrm{J}=6.5 \mathrm{~Hz}, 1.62 \mathrm{H}), 3.55(\mathrm{t}, \mathrm{J}=$ $6.6 \mathrm{~Hz}, 0.38 \mathrm{H}), 2.82(\mathrm{q}, \mathrm{J}=6.8 \mathrm{~Hz}, 1.65 \mathrm{H}), 2.62(\mathrm{q}, \mathrm{J}=6.8$ $\mathrm{Hz}, 0.48 \mathrm{H}), 1.38(\mathrm{~s}, 2.49 \mathrm{H}), 1.37(\mathrm{~s}, 6.63 \mathrm{H}) .{ }^{19} \mathbf{F}$ NMR $(282$ $\left.\mathrm{MHz}, \mathrm{CDCl}_{3}\right) \delta 84.2-80.8(\mathrm{~m}, 1 \mathrm{~F}), 66.3(\mathrm{dm}, \mathrm{J}=145.3$, $3.16 \mathrm{~F}), 64.4(\mathrm{dm}, \mathrm{J}=143.8,0.81 \mathrm{~F}) .{ }^{13} \mathbf{C}\left\{{ }^{1} \mathbf{H}\right\}$ NMR $(75 \mathrm{MHz}$, $\left.\mathrm{CDCl}_{3}\right) \delta 151.2,151.0,137.5,135.5,134.5,134.3(\mathrm{p}, \mathrm{J}=2.5$ $\mathrm{Hz}), 133.3$ (p, J = 2.6 Hz), 128.1, 126.2, 125.7, 125.5, 79.3 (p, $\mathrm{J}=12.3 \mathrm{~Hz}), 70.5(\mathrm{p}, \mathrm{J}=13.2 \mathrm{~Hz}), 43.6,43.4,34.7,34.7$, 32.5, 32.4, 31.4. HRMS (EI) m/z: M Calcd for $\mathrm{C}_{15} \mathrm{H}_{20} \mathrm{ClF}_{5} \mathrm{~S}$ 362.0894; Found 362.0899.

(Z)-(2-(2-(4-fluorophenyl)pent-5-chloropent-2-en-1-

yl)pentafluoro- $\lambda^{6}$-sulfane (Z-3g) and (E)-(2-(2-(4fluorophenyl)pent-5-chloropent-2-en-1-yl)pentafluoro- $\lambda^{6}$ -

sulfane (E-3g): Yellow liquid after column chromatography with petroleum ether / ethyl acetate $20: 1\left(\mathrm{R}_{\mathrm{f}}=0.2\right), 129.9 \mathrm{mg}$ (0.40 mmol), $80 \%$ yield, mixture of $\mathrm{E} / \mathrm{Z}$ isomers $3.6: 1 .{ }^{1} \mathbf{H}$ NMR $\left(300 \mathrm{MHz}, \mathrm{CDCl}_{3}\right) \delta 7.41-7.27(\mathrm{~m}, 1.60 \mathrm{H}), 7.25-$ $7.14(\mathrm{~m}, 0.51 \mathrm{H}), 7.08(\mathrm{td}, \mathrm{J}=8.7,7.0 \mathrm{~Hz}, 2 \mathrm{H}), 6.15-5.96(\mathrm{~m}$, $1 \mathrm{H}), 4.75(\mathrm{p}, \mathrm{J}=7.6 \mathrm{~Hz}, 1.59 \mathrm{H}), 4.58(\mathrm{p}, \mathrm{J}=7.6 \mathrm{~Hz}, 0.49 \mathrm{H})$, $3.68(\mathrm{t}, \mathrm{J}=6.4 \mathrm{~Hz}, 1.53 \mathrm{H}), 3.53(\mathrm{t}, \mathrm{J}=6.5 \mathrm{~Hz}, 0.49 \mathrm{H}), 2.79(\mathrm{q}$, $\mathrm{J}=6.7 \mathrm{~Hz}, 1.58 \mathrm{H}), 2.54(\mathrm{q}, \mathrm{J}=6.7 \mathrm{~Hz}, 0.52 \mathrm{H}) .{ }^{19} \mathbf{F}$ NMR $(282$ $\left.\mathrm{MHz}, \mathrm{CDCl}_{3}\right) \delta 86.5-77.8(\mathrm{~m}, 1 \mathrm{~F}), 66.4(\mathrm{dm}, \mathrm{J}=143.8 \mathrm{~Hz}$, $3.13 \mathrm{~F}), 64.6(\mathrm{dm}, \mathrm{J}=145.0 \mathrm{~Hz}, 0.86 \mathrm{~F}),-114.2,-114.7$. ${ }^{13} \mathbf{C}\left\{{ }^{1} \mathbf{H}\right\}$ NMR $\left(75 \mathrm{MHz}, \mathrm{CDCl}_{3}\right) \delta 162.6(\mathrm{~d}, \mathrm{~J}=247.4 \mathrm{~Hz})$, $162.4(\mathrm{~d}, \mathrm{~J}=247.6 \mathrm{~Hz}), 136.7,136.2,135.3,133.5$ (p, J = 2.5 $\mathrm{Hz}), 130.3$ (p, J = 2.6 Hz), 130.3 (d, J = 8.1 Hz), 128.3 (d, J = $8.1 \mathrm{~Hz}), 115.7(\mathrm{~d}, \mathrm{~J}=21.6 \mathrm{~Hz}), 115.7(\mathrm{~d}, \mathrm{~J}=21.6 \mathrm{~Hz}), 79.2(\mathrm{p}$, $\mathrm{J}=12.6 \mathrm{~Hz}), 70.6(\mathrm{p}, \mathrm{J}=13.5 \mathrm{~Hz}), 43.4,43.3$, 32.3. HRMS (EI) $\mathrm{m} / \mathrm{z}: \mathrm{M}^{+\bullet}$ Calcd for $\mathrm{C}_{11} \mathrm{H}_{11} \mathrm{ClF}_{6} \mathrm{~S}$ 324.0174; Found 324.0177 .

(Z)-(2-(2-(fluorophenyl)pent-5-chloropent-2-en-1-

yl)pentafluoro- $\lambda^{6}$-sulfane (Z-3h) and (E)-(2-(2(fluorophenyl)pent-5-chloropent-2-en-1-yl)pentafluoro- $\lambda^{6}$ -

sulfane (E-3h): Colorless oil after column chromatography with petroleum ether / ethyl acetate $20: 1\left(R_{\mathrm{f}}=0.2\right), 135.6 \mathrm{mg}$ (0.42 mmol), $83 \%$ yield, mixture of $\mathrm{E} / \mathrm{Z}$ isomers $1.9: 1 .{ }^{1} \mathbf{H}$ NMR $\left(300 \mathrm{MHz}, \mathrm{CDCl}_{3}\right) \delta 7.39-6.85(\mathrm{~m}, 4 \mathrm{H}), 6.04(\mathrm{t}, \mathrm{J}=$ $7.2 \mathrm{~Hz}, 0.36 \mathrm{H}), 5.93$ (t, J = 7.3 Hz, 0.62H), 4.71 (p, J = 8.0 Hz, $1.30 \mathrm{H}), 4.53(\mathrm{p}, \mathrm{J}=7.7 \mathrm{~Hz}, 0.72 \mathrm{H}), 3.57(\mathrm{t}, \mathrm{J}=6.5 \mathrm{~Hz}, 1.28$ $\mathrm{H}), 3.43(\mathrm{t}, \mathrm{J}=6.6 \mathrm{~Hz}, 0.72 \mathrm{H}), 2.70\left(\mathrm{q}_{\mathrm{appt}}, \mathrm{J}=6.7 \mathrm{~Hz}, 1.28 \mathrm{H}\right)$, $2.40\left(\mathrm{q}_{\mathrm{appt}}, \mathrm{J}=6.7 \mathrm{~Hz}, 0.72 \mathrm{H}\right) .{ }^{19} \mathbf{F}$ NMR $\left(282 \mathrm{MHz}, \mathrm{CDCl}_{3}\right) \delta$ $83.9-80.7(\mathrm{~m}, 1 \mathrm{~F}), 66.7(\mathrm{dm}, \mathrm{J}=144.6 \mathrm{~Hz}, 2.61 \mathrm{~F}), 65.0(\mathrm{dm}$, $\mathrm{J}=145.2 \mathrm{~Hz}, 1.40 \mathrm{~F}),-114.80,-116.55 .{ }^{13} \mathbf{C}\left\{{ }^{1} \mathbf{H}\right\} \mathbf{N M R}(75$ $\left.\mathrm{MHz}, \mathrm{CDCl}_{3}\right) \delta 159.9(\mathrm{~d}, \mathrm{~J}=246.4 \mathrm{~Hz}), 159.8(\mathrm{~d}, \mathrm{~J}=246.7$ $\mathrm{Hz}), 138.1,131.2$ (d, J = 3.6 Hz), 131.1 (d, J = 3.7 Hz), 130.2 $(\mathrm{d}, \mathrm{J}=8.4 \mathrm{~Hz}), 129.9(\mathrm{~d}, \mathrm{~J}=8.5 \mathrm{~Hz}), 129.6(\mathrm{t}, \mathrm{J}=2.9 \mathrm{~Hz})$, $128.9(\mathrm{t}, \mathrm{J}=2.7 \mathrm{~Hz}), 128.7,128.5,124.7(\mathrm{~d}, \mathrm{~J}=3.4 \mathrm{~Hz})$, 124.5, 124.4 (d, J = 3.5 Hz), $116.1(\mathrm{~d}, \mathrm{~J}=22.2 \mathrm{~Hz}), 115.8(\mathrm{~d}, \mathrm{~J}$ $=22.4 \mathrm{~Hz}), 79.30-77.83(\mathrm{~m}), 71.45-70.31(\mathrm{~m}), 43.2,43.0$, 32.6, 32.0. HRMS $\left(\mathrm{CI}^{+}\right) \mathrm{m} / \mathrm{z}: \mathrm{M}^{+\bullet}$ Calcd for $\mathrm{C}_{11} \mathrm{H}_{11} \mathrm{ClF}_{6} \mathrm{~S}$ 324.0174; Found 324.0170.

(Z)-(2-(4-(chlorophenyl)pent-5-chloropent-2-en-1-

yl)pentafluoro- $\lambda^{6}$-sulfane $\quad(\mathrm{Z}-3 \mathbf{i})$ and (E)-(2-(4(chlorophenyl)pent-5-chloropent-2-en-1-yl)pentafluoro- $\lambda^{6}$ -

sulfane (E-3i): Yellow liquid after column chromatography with petroleum ether / ethyl acetate $20: 1\left(R_{\mathrm{f}}=0.3\right), 150.3 \mathrm{mg}$ (0.44 mmol), $88 \%$ yield, mixture of $\mathrm{E} / \mathrm{Z}$ isomers $3.9: 1 .{ }^{1} \mathbf{H}$ 
NMR $\left(300 \mathrm{MHz}, \mathrm{CDCl}_{3}\right) \delta 7.44-7.32(\mathrm{~m}, 2 \mathrm{H}), 7.32-7.24$ (m, 1.55H), $7.20-7.11(\mathrm{~m}, 0.41 \mathrm{H}), 6.07(\mathrm{~m}, 1 \mathrm{H}), 4.74(\mathrm{p}, \mathrm{J}=$ $7.5 \mathrm{~Hz}, 1.58 \mathrm{H}), 4.58(\mathrm{p}, \mathrm{J}=7.6 \mathrm{~Hz}, 0.44 \mathrm{H}), 3.67(\mathrm{t}, \mathrm{J}=6.4 \mathrm{~Hz}$, $1.55 \mathrm{H}), 3.52(\mathrm{t}, \mathrm{J}=6.5 \mathrm{~Hz}, 0.43 \mathrm{H}), 2.79(\mathrm{q}, \mathrm{J}=6.7 \mathrm{~Hz}$, $1.57 \mathrm{H}), 2.54(\mathrm{q}, \mathrm{J}=6.7 \mathrm{~Hz}, 0.44 \mathrm{H}) .{ }^{19} \mathbf{F} \mathbf{N M R}(282 \mathrm{MHz}$, $\left.\mathrm{CDCl}_{3}\right) \delta 83.6-80.4(\mathrm{~m}, 1 \mathrm{~F}), 66.4(\mathrm{dm}, \mathrm{J}=145.3 \mathrm{~Hz}, 3.16 \mathrm{~F})$, $64.7(\mathrm{dm}, \mathrm{J}=146.7 \mathrm{~Hz}, 0.81 \mathrm{~F}) .{ }^{13} \mathbf{C}\left\{{ }^{1} \mathbf{H}\right\}$ NMR $(75 \mathrm{MHz}$, $\left.\mathrm{CDCl}_{3}\right) \delta 139.0,136.5,135.9,135.7,134.0,134.0,133.3(\mathrm{p}, \mathrm{J}$ $=2.4 \mathrm{~Hz}), 132.6(\mathrm{p}, \mathrm{J}=2.6 \mathrm{~Hz}), 129.9,128.9,127.9,79.0(\mathrm{t}, \mathrm{J}$ $=12.7 \mathrm{~Hz}), 70.3(\mathrm{p}, \mathrm{J}=13.6 \mathrm{~Hz}), 43.4,43.2,32.3$. HRMS (EI) $\mathrm{m} / \mathrm{z}$ : $\mathrm{M}^{+\bullet}$ Calcd for $\mathrm{C}_{11} \mathrm{H}_{11} \mathrm{Cl}_{2} \mathrm{~F}_{5} \mathrm{~S} 339.9879$; Found 339.9889.

(Z)-(2-(3-(chlorophenyl)pent-5-chloropent-2-en-1-

yl)pentafluoro- $\lambda^{6}$-sulfane $\quad(\mathrm{Z}-3 \mathbf{j}) \quad$ and $\quad(\mathrm{E})-(2-(3-$ (chlorophenyl)pent-5-chloropent-2-en-1-yl)pentafluoro- $\lambda^{6}$ -

sulfane (E-3j): Yellow liquid after column chromatography with petroleum ether / ethyl acetate $20: 1\left(R_{\mathrm{f}}=0.3\right), 150.3 \mathrm{mg}$ (0.44 mmol), $88 \%$ yield, mixture of $\mathrm{E} / \mathrm{Z}$ isomers $5.1: 1 .{ }^{1} \mathbf{H}$ NMR $\left(300 \mathrm{MHz}, \mathrm{CDCl}_{3}\right) \delta 7.30-7.17(\mathrm{~m}, 2.81 \mathrm{H}), 7.17-$ $7.07(\mathrm{~m}, 1.09 \mathrm{H}), 7.04-6.96(\mathrm{~m}, 0.18 \mathrm{H}), 5.98(\mathrm{~m}, 1 \mathrm{H}), 4.63$ $(\mathrm{p}, \mathrm{J}=7.5 \mathrm{~Hz}, 1.67 \mathrm{H}), 4.47(\mathrm{p}, \mathrm{J}=7.6 \mathrm{~Hz}, 0.38 \mathrm{H}), 3.57(\mathrm{t}, \mathrm{J}=$ $6.4 \mathrm{~Hz}, 1.66 \mathrm{H}), 3.42(\mathrm{t}, \mathrm{J}=6.5 \mathrm{~Hz}, 0.37 \mathrm{H}), 2.69(\mathrm{q}, \mathrm{J}=6.7 \mathrm{~Hz}$, $1.66 \mathrm{H}), 2.44(\mathrm{q}, \mathrm{J}=6.7 \mathrm{~Hz}, 0.38 \mathrm{H}) .{ }^{19} \mathbf{F}$ NMR $(282 \mathrm{MHz}$, $\left.\mathrm{CDCl}_{3}\right) \delta 83.4-80.1(\mathrm{~m}, 1 \mathrm{~F}), 66.5(\mathrm{dm}, \mathrm{J}=145.5 \mathrm{~Hz}, 3.32 \mathrm{~F})$, $64.7(\mathrm{dm}, \mathrm{J}=144.8 \mathrm{~Hz}, 0.65 \mathrm{~F}) .{ }^{13} \mathbf{C}\left\{{ }^{1} \mathbf{H}\right\}$ NMR $(75 \mathrm{MHz}$, $\left.\mathrm{CDCl}_{3}\right) \delta 142.4,139.3,136.8,136.4,134.7,134.5,133.2(\mathrm{p}, \mathrm{J}$ $=2.5 \mathrm{~Hz}), 132.6(\mathrm{p}, \mathrm{J}=2.7 \mathrm{~Hz}), 130.0,128.6,128.3,128.2$, 126.9, 126.8, 124.8, 78.9 (p, J = 12.8 Hz), 70.3 (p, J = 13.7 $\mathrm{Hz}$ ), 43.3, 43.1, 32.3. HRMS (EI) $\mathrm{m} / \mathrm{z}: \mathrm{M}^{+\bullet}$ Calcd for $\mathrm{C}_{11} \mathrm{H}_{11} \mathrm{Cl}_{2} \mathrm{~F}_{5} \mathrm{~S} 339.9879$; Found 339.9884.

(Z)-(2-(4-(bromophenyl)pent-5-chloropent-2-en-1-

yl)pentafluoro- $\lambda^{6}$-sulfane (Z-3k) and (E)-(2-(4(bromophenyl)pent-5-chloropent-2-en-1-yl)pentafluoro- $\lambda^{6}$ -

sulfane (E-3k): Colorless oil after column chromatography with petroleum ether / ethyl acetate $20: 1\left(\mathrm{R}_{\mathrm{f}}=0.3\right), 168.2 \mathrm{mg}$ (0.44 mmol), $87 \%$ yield, mixture of $\mathrm{E} / \mathrm{Z}$ isomers $3.7: 1 .{ }^{1} \mathbf{H}$ NMR $\left(300 \mathrm{MHz}, \mathrm{CDCl}_{3}\right) \delta 7.61-7.43(\mathrm{~m}, 2 \mathrm{H}), 7.29-7.19$ $(\mathrm{m}, 1.56 \mathrm{H}), 7.09(\mathrm{~d}, \mathrm{~J}=8.4 \mathrm{~Hz}, 0.43 \mathrm{H}), 6.07(\mathrm{dt}, \mathrm{J}=15.3,7.3$ $\mathrm{Hz}, 1 \mathrm{H}), 4.74(\mathrm{p}, \mathrm{J}=7.5 \mathrm{~Hz}, 1.57 \mathrm{H}), 4.57(\mathrm{p}, \mathrm{J}=7.6 \mathrm{~Hz}$, $0.45 \mathrm{H}), 3.67(\mathrm{t}, \mathrm{J}=6.4 \mathrm{~Hz}, 1.54 \mathrm{H}), 3.52(\mathrm{t}, \mathrm{J}=6.5 \mathrm{~Hz}, 0.54 \mathrm{H})$, $2.79\left(\mathrm{q}_{\text {appt }}, \mathrm{J}=6.7 \mathrm{~Hz}, 1.56 \mathrm{H}\right), 2.53\left(\mathrm{q}_{\mathrm{appt}}, \mathrm{J}=6.7 \mathrm{~Hz}, 0.46 \mathrm{H}\right)$. ${ }^{19}$ F NMR $\left(282 \mathrm{MHz}, \mathrm{CDCl}_{3}\right) \delta 84.5-80.5(\mathrm{~m}, 1 \mathrm{~F}), 67.0(\mathrm{dm}$, $\mathrm{J}=145.3 \mathrm{~Hz}, 3.14 \mathrm{~F}), 65.1(\mathrm{dm}, \mathrm{J}=143.3 \mathrm{~Hz}, 0.86 \mathrm{~F}) .{ }^{13} \mathbf{C}\left\{{ }^{1} \mathbf{H}\right\}$ NMR $\left(75 \mathrm{MHz}, \mathrm{CDCl}_{3}\right) \delta 139.5,136.5,136.4,135.8,133.4$ $(\mathrm{p}, \mathrm{J}=2.6 \mathrm{~Hz}), 132.6(\mathrm{p}, \mathrm{J}=3.0 \mathrm{~Hz}), 131.9,130.2,128.3$, 122.2, 78.9 (p, J = 12.7 Hz), 70.3 (p, J = 13.7 Hz), 43.3, 43.2, 32.3. HRMS $\left(\mathrm{CI}^{+}\right) \mathrm{m} / \mathrm{z}: \mathrm{M}^{+\bullet}$ Calcd for $\mathrm{C}_{11} \mathrm{H}_{11} \mathrm{BrClF}_{5} \mathrm{~S}$ 383.9374; Found 383.9384 .

(Z)-(5-chloro-2-(3,4-dichlorophenyl)pent-2-en-1-

yl)pentafluoro- $\lambda^{6}$-sulfane (Z-3I) and (Z)-(5-chloro-2-(3,4dichlorophenyl)pent-2-en-1-yl)pentafluoro- $\lambda^{6}$-sulfane (E-3l): Deviation from the general procedure: $1 \mathrm{mmol} \mathrm{SF}_{5} \mathrm{Cl}$ (2 equiv)

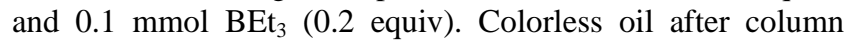
chromatography with petroleum ether / ethyl acetate 20:1 $\left(\mathrm{R}_{\mathrm{f}}\right.$ $=0.3), 167.1 \mathrm{mg}(0.45 \mathrm{mmol}), 89 \%$ yield, mixture of $\mathrm{E} / \mathrm{Z}$ isomers 4.4:1. ${ }^{1} \mathbf{H}$ NMR $\left(300 \mathrm{MHz}, \mathrm{CDCl}_{3}\right) \delta 7.54-7.39(\mathrm{~m}$, $1.86 \mathrm{H}), 7.31(\mathrm{~d}, \mathrm{~J}=2.1 \mathrm{~Hz}, 0.19 \mathrm{H}), 7.18(\mathrm{dd}, \mathrm{J}=8.4,2.2 \mathrm{~Hz}$, $0.82 \mathrm{H}), 7.06(\mathrm{dd}, \mathrm{J}=8.3,2.1 \mathrm{~Hz}, 0.19 \mathrm{H}), 6.09(\mathrm{~m}, 1 \mathrm{H}), 4.71$ $(\mathrm{p}, \mathrm{J}=7.5 \mathrm{~Hz}, 1.62 \mathrm{H}), 4.55(\mathrm{p}, \mathrm{J}=7.6 \mathrm{~Hz}, 0.39 \mathrm{H}), 3.68(\mathrm{t}, \mathrm{J}=$ $6.3 \mathrm{~Hz}, 1.61 \mathrm{H}), 3.53(\mathrm{t}, \mathrm{J}=6.4 \mathrm{~Hz}, 0.42 \mathrm{H}), 2.79(\mathrm{q}, \mathrm{J}=6.7 \mathrm{~Hz}$, $1.61 \mathrm{H}), 2.54(\mathrm{q}, \mathrm{J}=6.6 \mathrm{~Hz}, 0.40 \mathrm{H}) .{ }^{19} \mathbf{F} \mathbf{N M R}(282 \mathrm{MHz}$, $\left.\mathrm{CDCl}_{3}\right) \delta 83.4-80.4(\mathrm{~m}, 1 \mathrm{~F}), 66.5(\mathrm{dm}, \mathrm{J}=145.4,3.25 \mathrm{~F})$, $64.5(\mathrm{dm}, \mathrm{J}=144.7 \mathrm{~Hz}, 0.74 \mathrm{~F}) .{ }^{13} \mathbf{C}\left\{{ }^{1} \mathbf{H}\right\} \mathbf{N M R}(75 \mathrm{MHz}$,
$\left.\mathrm{CDCl}_{3}\right) \delta 140.6,137.5,137.4,136.8,132.9,132.9,132.4$, 132.2, 131.7 (p, J = 2.6 Hz), 130.7, 130.7, 130.4, 128.7, 128.0, 125.9, 78. 6 (p, $J=13.1 \mathrm{~Hz}), 70.1$ (p, $J=14.0 \mathrm{~Hz}), 43.2$, 43.0, 32.3. HRMS (EI) m/z: $\mathrm{M}^{+\bullet}$ Calcd for $\mathrm{C}_{11} \mathrm{H}_{10} \mathrm{Cl}_{3} \mathrm{~F}_{5} \mathrm{~S} 373.9489$; Found 373.9505.

(Z)-(5-chloro-2-(4-(trifluoromethyl)phenyl)pent-2-en-1-

yl)pentafluoro- $\lambda^{6}$-sulfane $(Z-3 m)$ and (E)-(5-chloro-2-(4(trifluoromethyl)phenyl)pent-2-en-1-yl)pentafluoro- $\lambda^{6}$-sulfane (E-3m): Colorless oil after column chromatography with petroleum ether / ethyl acetate $20: 1\left(\mathrm{R}_{\mathrm{f}}=0.3\right), 149.9 \mathrm{mg}(0.49$ mmol), 98\% yield, mixture of $\mathrm{E} / \mathrm{Z}$ isomers 5.1:1. ${ }^{1} \mathbf{H}$ NMR $\left(300 \mathrm{MHz}, \mathrm{CDCl}_{3}\right) \delta 7.75-7.58(\mathrm{~m}, 2 \mathrm{H}), 7.55-7.42(\mathrm{~m}$, $1.68 \mathrm{H}), 7.35(\mathrm{~d}, \mathrm{~J}=8.0 \mathrm{~Hz}, 0.37 \mathrm{H}), 6.17(\mathrm{t}, \mathrm{J}=7.3 \mathrm{~Hz}$, $0.81 \mathrm{H}), 6.11(\mathrm{t}, \mathrm{J}=7.3 \mathrm{~Hz}, 0.20 \mathrm{H}), 4.79(\mathrm{p}, \mathrm{J}=7.5 \mathrm{~Hz}$, $1.69 \mathrm{H}), 4.62(\mathrm{p}, \mathrm{J}=7.6 \mathrm{~Hz}, 0.38 \mathrm{H}), 3.69(\mathrm{t}, \mathrm{J}=6.4 \mathrm{~Hz}, 1.7 \mathrm{H})$, $3.54(\mathrm{t}, \mathrm{J}=6.4 \mathrm{~Hz}, 0.38 \mathrm{H}), 2.83(\mathrm{q}, \mathrm{J}=6.6 \mathrm{~Hz}, 1.68 \mathrm{H}), 2.54$ $(\mathrm{q}, \mathrm{J}=6.7 \mathrm{~Hz}, 0.36 \mathrm{H}) .{ }^{19} \mathbf{F}$ NMR $\left(282 \mathrm{MHz}, \mathrm{CDCl}_{3}\right) \delta 85.3-$ $78.4(\mathrm{~m}, 1 \mathrm{~F}), 66.5(\mathrm{dm}, \mathrm{J}=145.0 \mathrm{~Hz}, 3.35 \mathrm{~F}), 64.8(\mathrm{~d}, \mathrm{~J}=$ $144.0 \mathrm{~Hz}, 0.65 \mathrm{~F}) .{ }^{13} \mathrm{C}\left\{{ }^{1} \mathbf{H}\right\}$ NMR $\left(75 \mathrm{MHz}, \mathrm{CDCl}_{3}\right) \delta 144.2$, 141.3, 137.1, $133.3(\mathrm{p}, \mathrm{J}=2.5 \mathrm{~Hz}), 132.7(\mathrm{p}, \mathrm{J}=2.7 \mathrm{~Hz})$, $130.1(\mathrm{q}, \mathrm{J}=32.6 \mathrm{~Hz}), 130.2(\mathrm{q}, \mathrm{J}=32.5 \mathrm{~Hz}), 129.0,127.0$, 124.2 (q, J = 272.0 Hz), $124.2(\mathrm{q}, \mathrm{J}=272.0 \mathrm{~Hz}), 125.8(\mathrm{q}, \mathrm{J}=$ $3.8 \mathrm{~Hz}), 125.7$ (q, J = 3.8 Hz), 122.4, 122.4, 118.8, 118.8, 78.8 (p, J = 13.2 Hz), $70.1(\mathrm{p}, \mathrm{J}=14.0 \mathrm{~Hz}), 43.3,43.1,32.3,32.3$. HRMS (EI) m/z: ${ }^{+\bullet}$ Calcd for $\mathrm{C}_{12} \mathrm{H}_{11} \mathrm{Cl}_{1} \mathrm{~F}_{8} \mathrm{~S}$ 374.0142; Found 374.0154 .

(Z)-(2-([1,1'-biphenyl]-4-yl)-5-chloropent-2-en-1-

yl)pentafluoro- $\lambda^{6}$-sulfane (Z-3n) and (E)-(2-([1,1'-biphenyl]-4yl)-5-chloropent-2-en-1-yl)pentafluoro- $\lambda^{6}$-sulfane (E-3n): White solid after column chromatography with petroleum ether / ethyl acetate $20: 1\left(\mathrm{R}_{\mathrm{f}}=0.3\right), 164.6 \mathrm{mg}(0.43 \mathrm{mmol})$, $85 \%$ yield, mixture of $\mathrm{E} / \mathrm{Z}$ isomers $8.5: 1 .{ }^{1} \mathbf{H}$ NMR $(300 \mathrm{MHz}$, $\left.\mathrm{CDCl}_{3}\right) \delta 7.72-7.58(\mathrm{~m}, 4 \mathrm{H}), 7.57-7.36(\mathrm{~m}, 4.75 \mathrm{H}), 7.31(\mathrm{~d}$, $\mathrm{J}=8.3 \mathrm{~Hz}, 0.26 \mathrm{H}), 6.20(\mathrm{t}, \mathrm{J}=7.4 \mathrm{~Hz}, 0.87 \mathrm{H}), 6.09(\mathrm{t}, \mathrm{J}=7.1$ $\mathrm{Hz}, 0.14 \mathrm{H}), 4.84(\mathrm{p}, \mathrm{J}=7.6 \mathrm{~Hz}, 1.74 \mathrm{H}), 4.67(\mathrm{p}, \mathrm{J}=7.6 \mathrm{~Hz}$, $0.28 \mathrm{H}), 3.71(\mathrm{t}, \mathrm{J}=6.4 \mathrm{~Hz}, 1.73 \mathrm{H}), 3.57(\mathrm{t}, \mathrm{J}=6.6 \mathrm{~Hz}, 0.26 \mathrm{H})$, $2.84(\mathrm{q}, \mathrm{J}=6.7 \mathrm{~Hz}, 1.73 \mathrm{H}), 2.65(\mathrm{q}, \mathrm{J}=6.7 \mathrm{~Hz}, 0.28 \mathrm{H}) .{ }^{19} \mathbf{F}$ NMR $\left(282 \mathrm{MHz}, \mathrm{CDCl}_{3}\right) \delta 83.9-80.9(\mathrm{~m}, 1 \mathrm{~F}), 66.4(\mathrm{dm}, \mathrm{J}=$ $144.9 \mathrm{~Hz}, 3.58 \mathrm{~F}), 64.6(\mathrm{dm}, \mathrm{J}=145.2 \mathrm{~Hz}, 0.42 \mathrm{~F}) .{ }^{13} \mathbf{C}\left\{{ }^{1} \mathbf{H}\right\}$ NMR $\left(75 \mathrm{MHz}, \mathrm{CDCl}_{3}\right) \delta 140.9,140.8,140.5,140.5,139.3$, 136.3, 135.9, 135.1, 134.0 (p, J = 2.4 Hz), 133.1 (p, J = 2.7 $\mathrm{Hz}), 129.0,128.9,127.7,127.6,127.4,127.3,127.2,127.1$, 126.9, 79.2 (p, J = 12.5 Hz), 70.3 (p, J = 13.2 Hz), 43.6, 43.3, 32.4. HRMS (EI) $\mathrm{m} / \mathrm{z}$ : $\mathrm{M}^{+\bullet}$ Calcd for $\mathrm{C}_{17} \mathrm{H}_{16} \mathrm{ClF}_{5} \mathrm{~S} 382.0581$; Found 382.0566.

(Z)-(5-chloro-2-(naphthalen-2-yl)pent-2-en-1-yl)pentafluoro$\lambda^{6}$-sulfane (Z-3o) and (E)-(5-chloro-2-(naphthalen-2-yl)pent2-en-1-yl)pentafluoro- $\lambda^{6}$-sulfane (E-3o): White solid after column chromatography with petroleum ether / ethyl acetate $20: 1\left(\mathrm{R}_{\mathrm{f}}=0.3\right), 116.0 \mathrm{mg}(0.33 \mathrm{mmol}), 65 \%$ yield, mixture of $\mathrm{E} / \mathrm{Z}$ isomers $11.2: 1 .{ }^{1} \mathbf{H}$ NMR $\left(300 \mathrm{MHz} \mathrm{CDCl}_{3}\right) \delta 7.97-7.66$ (m, 4H), $7.63-7.30(\mathrm{~m}, 3 \mathrm{H}), 6.24(\mathrm{t}, \mathrm{J}=7.3 \mathrm{~Hz}, 0.89 \mathrm{H}), 6.12$ $(\mathrm{t}, \mathrm{J}=7.2 \mathrm{~Hz}, 0.10 \mathrm{H}), 4.90(\mathrm{p}, \mathrm{J}=7.6 \mathrm{~Hz}, 1.82 \mathrm{H}), 4.71(\mathrm{p}, \mathrm{J}=$ $7.7 \mathrm{~Hz}, 0.22 \mathrm{H}), 3.71(\mathrm{t}, \mathrm{J}=6.5 \mathrm{~Hz}, 1.82 \mathrm{H}), 3.53(\mathrm{t}, \mathrm{J}=6.5 \mathrm{~Hz}$, $0.22 \mathrm{H}), 2.86(\mathrm{q}, \mathrm{J}=6.7 \mathrm{~Hz}, 1.83 \mathrm{H}), 2.62(\mathrm{q}, \mathrm{J}=6.7 \mathrm{~Hz}$, $0.23 \mathrm{H}) .{ }^{19} \mathbf{F}$ NMR $\left(282 \mathrm{MHz}, \mathrm{CDCl}_{3}\right) \delta 83.8-80.1(\mathrm{~m}, 1 \mathrm{~F})$, $66.5(\mathrm{dm}, \mathrm{J}=143.0 \mathrm{~Hz}, 3.70 \mathrm{~F}), 64.1(\mathrm{dm}, \mathrm{J}=143.4 \mathrm{~Hz}$, 0.33F). ${ }^{13} \mathbf{C}\left\{{ }^{1} \mathbf{H}\right\}$ NMR (75 $\left.\mathrm{MHz}, \mathrm{CDCl}_{3}\right) \delta$ 137.8, 136.1, 135.6, 134.9, 134.9 (p, J = 2.2 Hz), 133.5 (p, J = 2.7 Hz), 133.4, 133.2, 133.0, 132.8, 128.4, 128.3, 128.2, 128.1, 127.8, $127.7,126.6,126.5,126.4,126.2,125.5,124.6,79.2$ (p, J = $12.2 \mathrm{~Hz}), 70.5$ (p, J = 12.2 Hz), 43.5, 43.3, 32.4, 32.4. HRMS 
(EI) $\mathrm{m} / \mathrm{z}: \mathrm{M}^{+\bullet}$ Calcd for $\mathrm{C}_{15} \mathrm{H}_{14} \mathrm{ClF}_{5} \mathrm{~S}$ 356.0425; Found 356.0418. A crystal was grown from a solution of $\mathbf{3 o}$ in DCM and petroleum ether by slow evaporation. X-Ray analysis is available from CCDC. ${ }^{22}{ }^{1} \mathrm{H}$ and ${ }^{19} \mathrm{~F}$ NMR data of pure Z-3o were recorded.

(Z)-(5-chloro-2-(naphthalen-1-yl)pent-2-en-1-yl)pentafluoro$\lambda^{6}$-sulfane (Z-3p) and (E)-(5-chloro-2-(naphthalen-1-yl)pent2-en-1-yl)pentafluoro- $\lambda^{6}$-sulfane (E-3p): Yellow oil after column chromatography with petroleum ether / ethyl acetate $20: 1\left(R_{\mathrm{f}}=0.3\right), 167.7 \mathrm{mg}(0.47 \mathrm{mmol}), 94 \%$ yield, mixture of E/Z isomers 4.0:1. ${ }^{1} \mathbf{H}$ NMR (300 $\left.\mathrm{MHz}, \mathrm{CDCl}_{3}\right) \delta 8.05-7.83$ $(\mathrm{m}, 2.25 \mathrm{H}), 7.80-7.68(\mathrm{~m}, 0.83 \mathrm{H}), 7.65-7.50(\mathrm{~m}, 3 \mathrm{H}), 7.44$ $(\mathrm{d}, \mathrm{J}=7.2 \mathrm{~Hz}, 1 \mathrm{H}), 6.41(\mathrm{t}, \mathrm{J}=7.1 \mathrm{~Hz}, 0.78 \mathrm{H}), 6.10(\mathrm{t}, \mathrm{J}=7.4$ $\mathrm{Hz}, 0.21 \mathrm{H}), 5.01-4.78(\mathrm{~m}, 1.17 \mathrm{H}), 4.80-4.59(\mathrm{~m}, 0.83 \mathrm{H})$, $3.73(\mathrm{t}, \mathrm{J}=6.3 \mathrm{~Hz}, 0.43 \mathrm{H}), 3.48(\mathrm{t}, \mathrm{J}=6.5 \mathrm{~Hz}, 1.57 \mathrm{H}), 2.95(\mathrm{q}$, $\mathrm{J}=6.7 \mathrm{~Hz}, 0.42 \mathrm{H}), 2.39(\mathrm{qd}, \mathrm{J}=6.7,3.7 \mathrm{~Hz}, 1.58 \mathrm{H}) .{ }^{19} \mathbf{F}$ NMR $\left(282 \mathrm{MHz}, \mathrm{CDCl}_{3}\right) \delta 84.3-79.8(\mathrm{~m}, 1 \mathrm{~F}), 66.8(\mathrm{dm}, \mathrm{J}=$ $143.2 \mathrm{~Hz}, 0.81 \mathrm{~F}), 65.1(\mathrm{dm}, \mathrm{J}=143.8 \mathrm{~Hz}, 3.22 \mathrm{~F}) .{ }^{13} \mathbf{C}\left\{{ }^{1} \mathbf{H}\right\}$ NMR $\left(75 \mathrm{MHz}, \mathrm{CDCl}_{3}\right) \delta 139.0,138.5,138.4,134.9,134.1$, 134.0, 132.58 (p, J = 2.4 Hz), $132.2(\mathrm{p}, \mathrm{J}=2.7 \mathrm{~Hz}), 131.0$, $130.7,129.0,128.9,128.7,128.5,127.1,126.7,126.6,126.5$, 126.1, 126.0, 125.5, 125.4, 124.9, 124.7, 79.1 (p, J = 12.7 Hz), 72.8 (p, J = 13.1 Hz), 43.3, 32.7, 32.3. HRMS (EI) m/z: $\mathbf{M}^{+\bullet}$ Calcd for $\mathrm{C}_{15} \mathrm{H}_{14} \mathrm{ClF}_{5} \mathrm{~S} 356.0425$; Found 356.0439.

(Z)-(2-benzyl-5-chloropent-2-en-1-yl)pentafluoro- $\lambda^{6}$-sulfane (Z-3q) and (E)-(2-benzyl-5-chloropent-2-en-1-yl)pentafluoro$\lambda^{6}$-sulfane (E-3q): Yellow oil after column chromatography with petroleum ether / ethyl acetate $20: 1\left(R_{\mathrm{f}}=0.4\right), 150.8 \mathrm{mg}$ $(0.47 \mathrm{mmol}), 94 \%$ yield, mixture of $\mathrm{E} / \mathrm{Z}$ isomers $2.7: 1 .{ }^{1} \mathbf{H}$ NMR $\left(300 \mathrm{MHz}, \mathrm{CDCl}_{3}\right) \delta 6.49-6.32(\mathrm{~m}, 3 \mathrm{H}), 6.27(\mathrm{td}, \mathrm{J}=$ 8.6, 7.9, $1.5 \mathrm{~Hz}, 2 \mathrm{H}), 4.97(\mathrm{t}, \mathrm{J}=7.1 \mathrm{~Hz}, 0.71 \mathrm{H}), 4.89(\mathrm{t}, \mathrm{J}=$ $7.3 \mathrm{~Hz}, 0.28 \mathrm{H}), 3.29$ (p, J = 7.5 Hz, 0.47H), 3.22 (p, J = 7.5 $\mathrm{Hz}, 1.54 \mathrm{H}), 2.79-2.64(\mathrm{~m}, 4 \mathrm{H}), 1.86(\mathrm{q}, \mathrm{J}=6.8 \mathrm{~Hz}, 1.43 \mathrm{H})$, $1.75(\mathrm{q}, \mathrm{J}=6.8 \mathrm{~Hz}, 0.56 \mathrm{H}) .{ }^{19} \mathbf{F} \mathbf{N M R}\left(282 \mathrm{MHz}, \mathrm{CDCl}_{3}\right) \delta$ $85.4-80.3(\mathrm{dm}, \mathrm{J}=142.2 \mathrm{~Hz}, 1 \mathrm{~F}), 66.4(\mathrm{dm}, \mathrm{J}=143.7 \mathrm{~Hz}$, 1.1F), $64.2(\mathrm{dm}, \mathrm{J}=143.7 \mathrm{~Hz}, 2.93 \mathrm{~F}) .{ }^{13} \mathbf{C}\left\{{ }^{1} \mathbf{H}\right\}$ NMR $(75$ $\left.\mathrm{MHz}, \mathrm{CDCl}_{3}\right) \delta 138.2,137.9,134.9,133.7,132.4(\mathrm{p}, \mathrm{J}=2.3$ $\mathrm{Hz}), 132.3$ (p, J = 2.3 Hz), 129.2, 129.0, 128.9, 128.8, 127.0, 126.9, 76.8 (p, J = 12.7 Hz), 70.0 (p, J = 13.5 Hz), 43.5, 43.4, 35.6, 31.7, 31.6. HRMS (EI) m/z: $\mathrm{M}^{+\bullet}$ Calcd for $\mathrm{C}_{15} \mathrm{H}_{14} \mathrm{ClF}_{5} \mathrm{~S}$ 320.0425 ; Found 320.0431.

(Z)-(5-chloro-2-phenethylpent-2-en-1-yl)pentafluoro- $\lambda^{6}$ -

sulfane (Z-3r) and (E)-(5-chloro-2-phenethylpent-2-en-1yl)pentafluoro- $\lambda^{6}$-sulfane (E-3r): Colorless oil after column chromatography with petroleum ether / ethyl acetate $20: 1\left(\mathrm{R}_{\mathrm{f}}\right.$ $=0.4), 162.4 \mathrm{mg}(0.49 \mathrm{mmol}), 97 \%$ yield, mixture of $\mathrm{E} / \mathrm{Z}$ isomers 4.1:1. ${ }^{1} \mathbf{H}$ NMR $\left(300 \mathrm{MHz}, \mathrm{CDCl}_{3}\right) \delta 6.17-6.04(\mathrm{~m}$, 2H), $6.04-5.88(\mathrm{~m}, 3 \mathrm{H}), 4.46(\mathrm{~m}, 1 \mathrm{H}), 3.06(\mathrm{p}, \mathrm{J}=8.0 \mathrm{~Hz}$, $0.41 \mathrm{H}), 2.93(\mathrm{p}, \mathrm{J}=8.1 \mathrm{~Hz}, 1.64 \mathrm{H}), 2.29(\mathrm{t}, \mathrm{J}=6.5 \mathrm{~Hz}$, $0.44 \mathrm{H}), 2.17(\mathrm{t}, \mathrm{J}=6.7 \mathrm{~Hz}, 1.58 \mathrm{H}), 1.63-1.44(\mathrm{~m}, 2 \mathrm{H}), 1.42$ $-1.16(\mathrm{~m}, 4 \mathrm{H}) .{ }^{19} \mathbf{F}$ NMR $\left(282 \mathrm{MHz}, \mathrm{CDCl}_{3}\right) \delta 84.6-81.0$ (m, 1F), $65.7(\mathrm{dm}, \mathrm{J}=142.3 \mathrm{~Hz}, 0.79 \mathrm{~F}), 63.9(\mathrm{dm}, \mathrm{J}=143.4$ $\mathrm{Hz}, 3.20 \mathrm{~F}) .{ }^{13} \mathbf{C}\left\{{ }^{1} \mathbf{H}\right\}$ NMR $\left(75 \mathrm{MHz}, \mathrm{CDCl}_{3}\right) \delta 140.9,140.8$, 134.8, $132.5(\mathrm{p}, \mathrm{J}=2.3 \mathrm{~Hz}), 131.9(\mathrm{p}, \mathrm{J}=2.5 \mathrm{~Hz}), 128.6$, $128.6,128.5,128.5,126.4,126.3,77.5(\mathrm{t}, \mathrm{J}=12.4 \mathrm{~Hz}), 71.0(\mathrm{t}$, $\mathrm{J}=13.1 \mathrm{~Hz}), 43.3,43.2,38.2$, 34.6, 34.2, 31.7, 31.5, 31.4. HRMS (EI) m/z: ${ }^{+\bullet}$ Calcd for $\mathrm{C}_{13} \mathrm{H}_{16} \mathrm{ClF}_{5} \mathrm{~S} 334.0581$; Found 334.0584

(Z)-(5-chloro-2-cyclohexylpent-2-en-1-yl)pentafluoro- $\lambda^{6}$ sulfane (Z-3s) and (E)-(5-chloro-2-cyclohexylpent-2-en-1yl)pentafluoro- $\lambda^{6}$-sulfane (E-3s): Colorless oil after column chromatography with petroleum ether / ethyl acetate $20: 1\left(R_{f}\right.$
$=0.4), 148.4 \mathrm{mg}(0.48 \mathrm{mmol}), 95 \%$ yield, mixture of $\mathrm{E} / \mathrm{Z}$ isomers 4.3:1. ${ }^{1} \mathbf{H}$ NMR $\left(300 \mathrm{MHz}, \mathrm{CDCl}_{3}\right) \delta 5.84-5.51(\mathrm{~m}$, $1 \mathrm{H}), 4.32(\mathrm{p}, \mathrm{J}=8.0 \mathrm{~Hz}, 1.53 \mathrm{H}), 4.21(\mathrm{p}, \mathrm{J}=8.1 \mathrm{~Hz}, 0.39 \mathrm{H})$, $3.63-3.45(\mathrm{~m}, 2 \mathrm{H}), 2.72-2.54(\mathrm{~m}, 2 \mathrm{H}), 2.49-2.34(\mathrm{~m}$, $0.21 \mathrm{H}), 2.02(\mathrm{~m}, 0.89 \mathrm{H}), 1.89-1.57(\mathrm{~m}, 5 \mathrm{H}), 1.39-1.04(\mathrm{~m}$, 5H). ${ }^{19}$ F NMR $\left(282 \mathrm{MHz}, \mathrm{CDCl}_{3}\right) \delta 85.2-80.3(\mathrm{~m}, 1 \mathrm{~F}), 65.7$ $(\mathrm{dm}, \mathrm{J}=142.9 \mathrm{~Hz}, 3.24 \mathrm{~F}), 63.5(\mathrm{dm}, \mathrm{J}=144.0 \mathrm{~Hz}, 0.75 \mathrm{~F})$. ${ }^{13} \mathbf{C}\left\{{ }^{1} \mathbf{H}\right\}$ NMR $\left(75 \mathrm{MHz}, \mathrm{CDCl}_{3}\right) \delta 138.4(\mathrm{p}, \mathrm{J}=2.1 \mathrm{~Hz})$, 137.6 (p, J = 1.9 Hz), 134.0, 129.5, $75.4(\mathrm{t}, \mathrm{J}=13.0 \mathrm{~Hz}), 71.3$ $(\mathrm{p}, \mathrm{J}=12.7 \mathrm{~Hz}), 43.9,43.6,43.6,40.8,32.8,31.6,31.5,31.1$, 26.9, 26.8, 26.3, 26.1. HRMS (EI) $\mathrm{m} / \mathrm{z}$ : $\mathbf{M}^{+\bullet}$ Calcd for $\mathrm{C}_{11} \mathrm{H}_{18} \mathrm{ClF}_{5} \mathrm{~S}$ 312.0738; Found 312.0732.

(Z)-(2-(3-chloropropylidene)decyl)pentafluoro- $\lambda^{6}$-sulfane (Z3t) and (E)-(2-(3-chloropropylidene)decyl)pentafluoro- $\lambda^{6}$ sulfane (E-3t): Yellow oil after column chromatography with petroleum ether / ethyl acetate $20: 1\left(R_{\mathrm{f}}=0.4\right), 157.3 \mathrm{mg}(0.46$ mmol), 92\% yield, mixture of $\mathrm{E} / \mathrm{Z}$ isomers $4.5: 1 .{ }^{1} \mathbf{H}$ NMR $\left(300 \mathrm{MHz}, \mathrm{CDCl}_{3}\right) \delta 5.66(\mathrm{t}, \mathrm{J}=7.1 \mathrm{~Hz}, 1 \mathrm{H}), 4.45-4.14(\mathrm{~m}$, $2 \mathrm{H}), 3.56(\mathrm{~m}, 2 \mathrm{H}), 2.59(\mathrm{~m}, 2 \mathrm{H}), 2.37-2.07(\mathrm{~m}, 2 \mathrm{H}), 1.45-$ $1.14(\mathrm{~m}, 12 \mathrm{H}), 1.01-0.63(\mathrm{~m}, 3 \mathrm{H}) .{ }^{19} \mathbf{F}$ NMR $(282 \mathrm{MHz}$, $\left.\mathrm{CDCl}_{3}\right) \delta 82.9(\mathrm{~m}, 1 \mathrm{~F}), 65.9(\mathrm{dm}, \mathrm{J}=142.5 \mathrm{~Hz}, 0.73 \mathrm{~F}), 63.7$ $(\mathrm{dm}, \mathrm{J}=143.3,3.25 \mathrm{~F}) .{ }^{13} \mathbf{C}\left\{{ }^{1} \mathbf{H}\right\} \mathbf{N M R}\left(75 \mathrm{MHz}, \mathrm{CDCl}_{3}\right) \delta$. 133.9 (p, J = 2.2 Hz), 133.7, 132.9 (p, J = 2.1 Hz), 77.6 (p, J = $12.5 \mathrm{~Hz}), 71.0(\mathrm{p}, \mathrm{J}=13.1 \mathrm{~Hz}), 43.5,43.4,36.6,32.0,31.6$, 31.5, 29.8, 29.6, 29.5, 29.4, 29.4, 29.3, 29.2, 28.2, 28.0, 22.8, 14.2. HRMS (EI) $\mathrm{m} / \mathrm{z}: \mathrm{M}^{+}$Calcd for $\mathrm{C}_{13} \mathrm{H}_{24} \mathrm{ClF}_{5} \mathrm{~S} 342.1207$; Found 342.1214.

(Z)-(2-(3-chloropropylidene)decyl)pentafluoro- $\lambda^{6}$-sulfane (Z3u) and (E)-(2-(3-chloropropylidene)decyl)pentafluoro- $\lambda^{6}$ sulfane (E-3u): Yellow oil after column chromatography with petroleum ether / ethyl acetate $20: 1\left(R_{\mathrm{f}}=0.4\right), 172.3 \mathrm{mg}(0.45$ mmol), $90 \%$ yield, mixture of $\mathrm{E} / \mathrm{Z}$ isomers $>20: 1 .{ }^{1} \mathbf{H}$ NMR $\left(300 \mathrm{MHz}, \mathrm{CDCl}_{3}\right) \delta 7.65-7.27(\mathrm{~m}, 9.76 \mathrm{H}), 7.16(\mathrm{~m}, 0.16 \mathrm{H})$, $6.18(\mathrm{t}, \mathrm{J}=7.4 \mathrm{~Hz}, 0.91 \mathrm{H}), 6.08(\mathrm{t}, \mathrm{J}=7.2 \mathrm{~Hz}, 0.06 \mathrm{H}), 5.10$ $(\mathrm{dd}, \mathrm{J}=8.1,5.9 \mathrm{~Hz}, 0.91 \mathrm{H}), 4.97(\mathrm{dd}, \mathrm{J}=7.7,6.3 \mathrm{~Hz}, 0.08 \mathrm{H})$, 4.76 (p, J = 7.5 Hz, 1.85H), 4.62 (p, J = 7.6 Hz, 0.12H), $3.38-$ $3.02(\mathrm{~m}, 1.85 \mathrm{H}), 3.02-2.82(\mathrm{~m}, 0.13 \mathrm{H}) .{ }^{19} \mathbf{F}$ NMR $(282 \mathrm{MHz}$, $\left.\mathrm{CDCl}_{3}\right) \delta 84.3-80.4(\mathrm{~m}, 1 \mathrm{~F}), 66.5(\mathrm{dm}, \mathrm{J}=145.1 \mathrm{~Hz}, 3.86 \mathrm{~F})$, $64.7(\mathrm{dm}, \mathrm{J}=146.5 \mathrm{~Hz}, 0.15 \mathrm{~F}) .{ }^{13} \mathbf{C}\left\{{ }^{1} \mathbf{H}\right\}$ NMR $(75 \mathrm{MHz}$, $\left.\mathrm{CDCl}_{3}\right) \delta 140.8,140.7,140.6,137.5,135.2,134.6,133.6(\mathrm{p}, \mathrm{J}$ $=2.6 \mathrm{~Hz}), 128.9,128.9,128.8,128.7,128.6,128.6,128.5$, $128.4,128.3,128.1,128.0,79.3(\mathrm{~d}, \mathrm{~J}=13.1 \mathrm{~Hz}), 70.5$ (p, J = 13.3 Hz), 62.3, 62.1, 39.8, 39.6. HRMS $\left(\mathrm{CI}^{+}\right) \mathrm{m} / \mathrm{z}: \mathrm{M}^{+\bullet} \mathrm{Calcd}$ for $\mathrm{C}_{17} \mathrm{H}_{16} \mathrm{ClF}_{5} \mathrm{~S}$ 382.0581; Found 382.0580.

(Z)-(5-chloro-2-(4-methoxyphenyl)-5-phenylpent-2-en-1-

yl)pentafluoro- $\lambda^{6}$-sulfane (Z-3v) and (E)-(5-chloro-2-(4methoxyphenyl)-5-phenylpent-2-en-1-yl)pentafluoro- $\lambda^{6}$ -

sulfane (E-3v): Yellow oil after column chromatography with petroleum ether / ethyl acetate $20: 1\left(R_{\mathrm{f}}=0.4\right), 156.0 \mathrm{mg}(0.38$ $\mathrm{mmol}), 76 \%$ yield, mixture of $\mathrm{E} / \mathrm{Z}$ isomers $15.8: 1 .{ }^{1} \mathbf{H}$ NMR $\left(300 \mathrm{MHz}, \mathrm{CDCl}_{3}\right) \delta 7.41-7.16(\mathrm{~m}, 5 \mathrm{H}), 7.15-7.05(\mathrm{~m}$, $1.86 \mathrm{H}), 6.89(\mathrm{~d}, \mathrm{~J}=8.7 \mathrm{~Hz}, 0.17 \mathrm{H}), 6.83-6.67(\mathrm{~m}, 2 \mathrm{H}), 5.90$ $(\mathrm{t}, \mathrm{J}=7.4 \mathrm{~Hz}, 0.89 \mathrm{H}), 5.83(\mathrm{t}, \mathrm{J}=7.1 \mathrm{~Hz}, 0.08 \mathrm{H}), 4.44-4.31$ $(\mathrm{m}, 0.91 \mathrm{H}), 4.82-4.71(\mathrm{~m}, 0.09 \mathrm{H}), 4.64-4.44(\mathrm{~m}, 1.82 \mathrm{H})$, $4.38(\mathrm{q}, \mathrm{J}=7.7 \mathrm{~Hz}, 0.16 \mathrm{H}), 3.67(\mathrm{~s}, 0.45 \mathrm{H}), 3.67(\mathrm{~s}, 2.50 \mathrm{H})$, $3.08-2.78(\mathrm{~m}, 1.84 \mathrm{H}), 2.81-2.62(\mathrm{~m}, 0.16 \mathrm{H}) .{ }^{19} \mathbf{F}$ NMR $(282$ $\left.\mathrm{MHz} \mathrm{CDCl}_{3}\right) \delta 85.7-79.8(\mathrm{~m}, 1 \mathrm{~F}), 66.5(\mathrm{dm}, \mathrm{J}=143.3 \mathrm{~Hz}$, $0.24 \mathrm{~F}), 64.6(\mathrm{dm}, \mathrm{J}=144.9 \mathrm{~Hz}, 0.24 \mathrm{~F}) .{ }^{13} \mathbf{C}\left\{{ }^{\mathbf{1}} \mathbf{H}\right\}$ NMR $(75$ $\left.\mathrm{MHz}, \mathrm{CDCl}_{3}\right) \delta 159.6,159.2,140.8,140.8,134.7,134.1(\mathrm{p}, \mathrm{J}$ $=2.6 \mathrm{~Hz}), 133.2,132.9(\mathrm{p}, \mathrm{J}=2.6 \mathrm{~Hz}), 129.6,128.9,128.7$, 128.6, 127.7, 127.0, 127.0, 114.1, 114.0, 79.5 (p, J = 12.3 Hz), $70.6(\mathrm{p}, \mathrm{J}=13.0 \mathrm{~Hz}), 62.3,62.2,55.3,55.2,39.7,39.6$. 
HRMS (EI) m/z: $\mathrm{M}^{+\cdot}$ Calcd for $\mathrm{C}_{18} \mathrm{H}_{18} \mathrm{ClF}_{5} \mathrm{OS}$ 412.0687; Found 412.0692 .

(Z)-(5-chloro-5-phenyl-2-(4-(trifluoromethyl)phenyl)pent-2en-1-yl)pentafluoro- $\lambda^{6}$-sulfane $(Z-3 w)$ and (E)-(5-chloro-5phenyl-2-(4-(trifluoromethyl)phenyl)pent-2-en-1-

yl)pentafluoro- $\lambda^{6}$-sulfane (E-3w): Colorless oil after column chromatography with petroleum ether / ethyl acetate $20: 1\left(R_{f}\right.$ $=0.4), 190.6 \mathrm{mg}(0.43 \mathrm{mmol}), 85 \%$ yield, mixture of $\mathrm{E} / \mathrm{Z}$ isomers > 20:1. ${ }^{1} \mathbf{H}$ NMR $\left(300 \mathrm{MHz}, \mathrm{CDCl}_{3}\right) \delta 7.66(\mathrm{~d}, \mathrm{~J}=8.2$ $\mathrm{Hz}, 2 \mathrm{H}), 7.53-7.28(\mathrm{~m}, 7 \mathrm{H}), 6.30-6.07$ (m, 1H), 5.09 (dd, J $=8.1,5.8 \mathrm{~Hz}, 0.96 \mathrm{H}), 4.97(\mathrm{dd}, \mathrm{J}=7.5,6.3 \mathrm{~Hz}, 0.05 \mathrm{H}), 4.85-$ $4.62(\mathrm{~m}, 1.93 \mathrm{H}), 4.64-4.45(\mathrm{~m}, 0.09 \mathrm{H}), 3.30-2.98(\mathrm{~m}$, $1.94 \mathrm{H}), 2.96-2.76(\mathrm{~m}, 0.08 \mathrm{H}) .{ }^{19} \mathbf{F}$ NMR $\left(282 \mathrm{MHz}, \mathrm{CDCl}_{3}\right)$ $\delta 83.7-80.7(\mathrm{~m}, 1 \mathrm{~F}), 66.7(\mathrm{dm}, \mathrm{J}=142.8 \mathrm{~Hz}, 3.96 \mathrm{~F}), 65.4$ $(\mathrm{dd}, \mathrm{J}=143.8 \mathrm{~Hz}, 0.08 \mathrm{~F}),-63.0,-63.1 .{ }^{13} \mathbf{C}\left\{{ }^{1} \mathbf{H}\right\}$ NMR $(75$ $\left.\mathrm{MHz}, \mathrm{CDCl}_{3}\right) \delta 144.1,140.6,136.4,132.7(\mathrm{t}, \mathrm{J}=2.7 \mathrm{~Hz})$, 130.2 (q, J = 32.6 Hz), 129.0, 128.9, 127.0, 127.0, 125.7 (q, J = 3.8 Hz), $124.2(\mathrm{~d}, \mathrm{~J}=272.0 \mathrm{~Hz}), 70.1(\mathrm{p}, \mathrm{J}=14.0 \mathrm{~Hz}), 61.8$, 39.8. HRMS (EI) $\mathrm{m} / \mathrm{z}: \mathrm{M}^{+\bullet}$ Calcd for $\mathrm{C}_{18} \mathrm{H}_{15} \mathrm{ClF}_{8} \mathrm{~S} 450.0455$; Found 450.0461 .

(Z)-(5-chloro-2-phenyl-5-(4-(trifluoromethyl)phenyl)pent-2en-1-yl)pentafluoro- $\lambda^{6}$-sulfane $(Z-3 \mathbf{x})$ and (E)-(5-chloro-5phenyl-2-(4-(trifluoromethyl)phenyl)pent-2-en-1-

yl)pentafluoro- $\lambda^{6}$-sulfane (E-3x): Colorless oil after column chromatography with petroleum ether / ethyl acetate $20: 1\left(\mathrm{R}_{\mathrm{f}}\right.$ $=0.4), 182.4 .6 \mathrm{mg}(0.41 \mathrm{mmol}), 81 \%$ yield, mixture of $\mathrm{E} / \mathrm{Z}$ isomers 3.6:1. ${ }^{\mathbf{1}} \mathbf{H}$ NMR $\left(300 \mathrm{MHz}, \mathrm{CDCl}_{3}\right) \delta 7.71(\mathrm{~d}, \mathrm{~J}=8.1$ $\mathrm{Hz}, 1.58 \mathrm{H}), 7.59$ (d, J = 8.0 Hz, 2H), $7.38(\mathrm{~m}, 5 \mathrm{H}), 7.19-6.97$ $(\mathrm{m}, 0.49 \mathrm{H}), 6.13(\mathrm{t}, \mathrm{J}=7.3 \mathrm{~Hz}, 0.75 \mathrm{H}), 6.04(\mathrm{t}, \mathrm{J}=7.2 \mathrm{~Hz}$, $0.22 \mathrm{H}), 5.09(\mathrm{dd}, \mathrm{J}=8.2,5.7 \mathrm{~Hz}, 0.73 \mathrm{H}), 4.95(\mathrm{t}, \mathrm{J}=7.0 \mathrm{~Hz}$, $0.25 \mathrm{H}), 4.76(\mathrm{p}, \mathrm{J}=7.5 \mathrm{~Hz}, 1.49 \mathrm{H}), 4.59(\mathrm{p}, \mathrm{J}=7.6 \mathrm{~Hz}$, $0.47 \mathrm{H}), 3.25-2.99(\mathrm{~m}, 1.48 \mathrm{H}), 3.01-2.76(\mathrm{~m}, 0.52 \mathrm{H}) .{ }^{19} \mathbf{F}$ NMR $\left(282 \mathrm{MHz}, \mathrm{CDCl}_{3}\right) \delta 146.2-142.7(\mathrm{~m}), 129.0(\mathrm{dm}, \mathrm{J}=$ $143.4 \mathrm{~Hz}, 3.11 \mathrm{~F}), 127.1(\mathrm{dm}, \mathrm{J}=144.3 \mathrm{~Hz}, 0.86 \mathrm{~F}),-63.1$. ${ }^{13} \mathbf{C}\left\{{ }^{1} \mathbf{H}\right\}$ NMR $\left(75 \mathrm{MHz}, \mathrm{CDCl}_{3}\right) \delta 144.7(\mathrm{q}, \mathrm{J}=1.4 \mathrm{~Hz})$, $144.6(\mathrm{q}, \mathrm{J}=1.4 \mathrm{~Hz}), 140.4,137.3,135.2(\mathrm{p}, \mathrm{J}=2.5 \mathrm{~Hz})$, 134.3, 134.1 (p, J = 2.7 Hz), 133.9, $131.0(\mathrm{q}, \mathrm{J}=32.6 \mathrm{~Hz})$, $130.7(q, J=32.6 \mathrm{~Hz}), 128.8,128.7,128.4,128.3,128.1$, $127.5,126.6,126.0(\mathrm{q}, \mathrm{J}=3.8 \mathrm{~Hz}), 125.8(\mathrm{q}, \mathrm{J}=3.5 \mathrm{~Hz})$, $124.0(\mathrm{~d}, \mathrm{~J}=272.3 \mathrm{~Hz}), 118.6,79.2(\mathrm{p}, \mathrm{J}=12.7 \mathrm{~Hz}), 70.5(\mathrm{p}, \mathrm{J}$ $=13.5 \mathrm{~Hz}), 61.0(\mathrm{p}, \mathrm{J}=4.5 \mathrm{~Hz}), 39.6,39.5$. HRMS (EI) m/z: $\mathrm{M}^{+\bullet}$ Calcd for $\mathrm{C}_{18} \mathrm{H}_{15} \mathrm{ClF}_{8} \mathrm{~S} 450.0455$; Found 450.0464.

(Z)-(2-(tert-butyl)-5-chloro-5-phenylpent-2-en-1-

yl)pentafluoro- $\lambda^{6}$-sulfane (Z-3y) and (E)- -(2-(tert-butyl)-5chloro-5-phenylpent-2-en-1-yl)pentafluoro- $\lambda^{6}$-sulfane (E-3y): Colorless oil after column chromatography with petroleum ether / ethyl acetate 30:1 $\left(\mathrm{R}_{\mathrm{f}}=0.6\right), 169.7 \mathrm{mg}(0.47 \mathrm{mmol})$, $94 \%$ yield, mixture of E/Z isomers > 100:1. ${ }^{1} \mathbf{H}$ NMR $(300$ $\left.\mathrm{MHz} \mathrm{CDCl}_{3}\right) \delta 7.51-6.95(\mathrm{~m}, 5 \mathrm{H}), 5.81(\mathrm{t}, \mathrm{J}=7.2 \mathrm{~Hz}, 1 \mathrm{H})$, $4.82(\mathrm{dd}, \mathrm{J}=8.1,5.9 \mathrm{~Hz}, 1 \mathrm{H}), 4.23(\mathrm{pd}, \mathrm{J}=7.8,2.2 \mathrm{~Hz}, 2 \mathrm{H})$, $3.05-2.58(\mathrm{~m}, 2 \mathrm{H}), 1.00(\mathrm{~s}, 9 \mathrm{H}) .{ }^{19} \mathbf{F}$ NMR $(282 \mathrm{MHz}$, $\left.\mathrm{CDCl}_{3}\right) \delta 85.4-81.2(\mathrm{~m}, 1 \mathrm{~F}), 65.9(\mathrm{dm}, \mathrm{J}=143.1 \mathrm{~Hz}, 4 \mathrm{~F})$. ${ }^{13} \mathbf{C}\left\{{ }^{1} \mathbf{H}\right\}$ NMR $\left(75 \mathrm{MHz}, \mathrm{CDCl}_{3}\right) \delta 141.2,140.5(\mathrm{p}, \mathrm{J}=1.9$ $\mathrm{Hz}), 131.4,128.8,128.6,127.0,69.6$ (p, J = 13.7 Hz), 62.6, 39.6 (p, J = 2.2 Hz), 36.8, 30.3. HRMS (EI) m/z: $\mathbf{M}^{+\bullet}$ Calcd for $\mathrm{C}_{15} \mathrm{H}_{20} \mathrm{ClF}_{5} \mathrm{~S}$ 362.0894; Found 362.0898.

(2-chloro-2-cyclobutyl-2-phenylethyl)pentafluoro- $\lambda^{6}$-sulfane (6): Colorless oil after column chromatography with petroleum ether/ethyl acetate $20: 1\left(\mathrm{R}_{\mathrm{f}}=0.6\right), 91.4 \mathrm{mg}(0.29 \mathrm{mmol})$, $57 \%$ yield. ${ }^{1} \mathbf{H}$ NMR $\left(300 \mathrm{MHz}, \mathrm{CDCl}_{3}\right) \delta 7.57-7.46(\mathrm{~m}, 2 \mathrm{H})$, $7.45-7.27$ (m, 3H), $4.36(\mathrm{pd}, J=8.2,3.7 \mathrm{~Hz}, 2 \mathrm{H}), 3.51-3.14$ (m, 1H), $2.50-2.00(\mathrm{~m}, 3 \mathrm{H}), 2.02-1.71(\mathrm{~m}, 3 \mathrm{H}) .{ }^{19} \mathbf{F}$ NMR $\left(282 \mathrm{MHz}, \mathrm{CDCl}_{3}\right) \delta 87.1-79.4(\mathrm{~m}, 1 \mathrm{~F}), 69.9(\mathrm{dm}, J=148.1$ $\mathrm{Hz}, 4 \mathrm{~F}) .{ }^{13} \mathbf{C}\left\{{ }^{1} \mathbf{H}\right\}$ NMR (75 $\left.\mathbf{M H z}, \mathrm{CDCl}_{3}\right) \delta 139.4,128.3$, 128.2, 126.8, 78.7 (p, $J=11.1 \mathrm{~Hz}$ ), 44.1, 24.9, 23.9, 16.6. HRMS $\left(\mathrm{CI}^{+}\right) \mathrm{m} / \mathrm{z}: \mathrm{M}^{+}$Calcd for $\mathrm{C}_{12} \mathrm{H}_{14} \mathrm{ClF}_{5} \mathrm{~S}$ 320.0425; Found 320.0420 .

\section{Ozonolysis: Access to $\boldsymbol{\alpha}-\mathrm{SF}_{\mathbf{5}}$ ketones. $^{28}$}

Typical experiment: Compound 3a (123.1 mg, $0.5 \mathrm{mmol}$, 1equiv) was dissolved in ethyl acetate $(20 \mathrm{~mL})$, and the solution was cooled to $-60{ }^{\circ} \mathrm{C}$. The solution was sparged with ozone for $10 \mathrm{~min}$, and sparged with nitrogen for $10 \mathrm{~min}$. Dimethylsulfide (10 mmol, 20 equiv) was added, and the reaction was allowed to stir for 10 hours while warming gradually to room temperature. The solvent was removed in vacuo. The ketone compound was purified by flash silica chromatography $(\mathrm{Rf}=0.25$, petroleum ether/ethyl acetate $20: 1)$ to afford the product 7a quantitatively as evaluated by ${ }^{19} \mathrm{~F}$ NMR. However, due to high volatility of $\mathbf{7 a}$, yields vary in the range 50 $85 \%$ in repeated experiments.

All 2-(pentafluoro- $\lambda^{6}$-sulfanyl) ketones 7 a-d are known compounds for which analytical data are identical to those reported in the literature. 7a CAS [129421-48-3], ${ }^{24 \mathrm{e}} \mathbf{7 b}$ CAS [231032534-7], ${ }^{24 \mathrm{e}}$ 7c CAS [2669730-32-7], ${ }^{24 \mathrm{f}}$ 7d CAS [2310325-24$5] .^{24 \mathrm{e}}$

\section{ASSOCIATED CONTENT}

\section{Supporting Information}

The material is available free of charge via the Internet at http://pubs.acs.org.

Copies of ${ }^{1} \mathrm{H},{ }^{19} \mathrm{~F}$ and ${ }^{13} \mathrm{C}\left\{{ }^{1} \mathrm{H}\right\}$ NMR spectra of the products (pdf).

\section{AUTHOR INFORMATION}

\section{Corresponding Authors}

*majun_an68@tju.edu.cn

* dominique.cahard@univ-rouen.fr

\section{ORCID}

Jun-An Ma: 0000-0002-3902-6799

Dominique Cahard: 0000-0002-8510-1315

\section{Author Contributions}

The manuscript was written through contributions of all authors. All authors have given approval to the final version of the manuscript.

\section{ACKNOWLEDGMENT}

Our research was supported by the Centre National de la Recherche Scientifique CNRS, Normandy University, Labex SynOrg (ANR-11-LABX-0029), the graduate school for research XLChem (ANR-18-EURE-0020 XL CHEM) and the Région Normandie. F-F. F. is grateful to the French ministry of foreign affairs for an Eiffel excellence doctorate scholarship.

\section{REFERENCES}

(1) (a) Wang, J.; Blaszczyk, S. A.; Li, X.; Tang, W., Transition Metal-Catalyzed Selective Carbon-Carbon Bond Cleavage of Vinylcyclopropanes in Cycloaddition Reactions. Chem. Rev. 2021, 121, 110-139. (b) Schneider, T. F.; Kaschel, J.; Werz, D. B., A New Golden Age for Donor-Acceptor Cyclopropanes. Angew. Chem. Int. Ed. 2014, 53, 5504-5523. (c) Ganesh, V.; Chandrasekaran, S., Recent Advances in the Synthesis and Reactivity of Vinylcyclopropanes. 
Synthesis 2016, 48, 4347-4380. (d) Jiao, L.; Yu, Z.-X. Vinylcyclopropane Derivatives in Transition-Metal-Catalyzed Cycloadditions for the Synthesis of Carbocyclic Compounds. J. Org. Chem. 2013, 78, 6842-6848.

(2) (a) Sherry, B. D.; Fürstner, A., Iron-catalyzed addition of Grignard reagents to activated vinyl cyclopropanes. Chem. Commun. 2009, 7116-7118. (b) Moran, J.; Smith, A. G.; Carris, R. M.; Johnson, J. S.; Krische, M. J., Polarity Inversion of Donor-Acceptor Cyclopropanes: Disubstituted $\delta$-Lactones via Enantioselective Iridium Catalysis. J. Am. Chem. Soc. 2011, 133, 18618-18621. (c) Dieskau, A. P.; Holzwarth, M. S.; Plietker, B., Fe-Catalyzed Allylic C-C-Bond Activation: Vinylcyclopropanes as Versatile a1, a3, d5-Synthons in Traceless Allylic Substitutions and [3+2]-Cycloadditions. J. Am. Chem. Soc. 2012, 134, 5048-5051. (d) Sumida, Y.; Yorimitsu, H.; Oshima, K., Nickel-Catalyzed Borylative Ring-Opening Reaction of Vinylcyclopropanes with Bis(pinacolato)diboron Yielding Allylic Boronates. Org. Lett. 2008, 10, 4677-4679.

(3) (a) Wender, P. A.; Takahashi, H.; Witulski, B., Transition Metal Catalyzed [5 + 2] Cycloadditions of Vinylcyclopropanes and Alkynes: A Homolog of the Diels-Alder Reaction for the Synthesis of Seven-Membered Rings. J. Am. Chem. Soc. 1995, 117, 4720-4721. (b) Caillé, J.; Robiette, R., Cycloaddition of cyclopropanes for the elaboration of medium-sized carbocycles. Org. Biomol. Chem. 2021, $19,5702-5724$

(4) Suginome, M.; Matsuda, T.; Yoshimoto, T.; Ito, Y., NickelCatalyzed Silaboration of Small-Ring Vinylcycloalkanes: Regio- and Stereoselective (E)-Allylsilane Formation via $\mathrm{C}-\mathrm{C}$ Bond Cleavage. Organometallics 2002, 21, 1537-1539.

(5) Chen, C.; Shen, X.; Chen, J.; Hong, X.; Lu, Z., IronCatalyzed Hydroboration of Vinylcyclopropanes. Org. Lett. 2017, 19, $5422-5425$.

(6) Liu, L.; Lee, W.; Yuan, M.; Acha, C.; Geherty, M. B.; Williams, B.; Gutierrez, O., Intra- and intermolecular Fe-catalyzed dicarbofunctionalization of vinyl cyclopropanes. Chem. Sci. 2020, 11, 3146-3151.

(7) Combee, L. A.; Johnson, S. L.; Laudenschlager, J. E.; Hilinski, M. K., Rh(II)-Catalyzed Nitrene-Transfer $[5+1]$ Cycloadditions of Aryl-Substituted Vinylcyclopropanes. Org. Lett. 2019, 21, 2307-2311.

(8) Zhang, Z.-Q.; Meng, X.-Y.; Sheng, J.; Lan, Q.; Wang, X.S., Enantioselective Copper-Catalyzed 1,5-Cyanotrifluoromethylation of Vinylcyclopropanes. Org. Lett. 2019, 21, 8256-8260.

(9) Shi, W.-J.; Liu, Y.; Butti, P.; Togni, A., Gold(I)- and Brønsted Acid-Catalyzed Ring-Opening of Unactivated Vinylcyclopropanes with Sulfonamides. Adv. Synth. Catal. 2007, 349, $1619-1623$.

(10) Li Jin, C. J., Huang Wenhao, Cheng Xu, E-Z Isomerization of 1,5-Bromotrichloromethylation Reaction Products and Trisubstituted Styrenes. Chin. J. Org. Chem. 2018, 38, 1507-1515.

(11) Long, P.-W.; He, T.; Oestreich, M., B $\left(\mathrm{C}_{6} \mathrm{~F}_{5}\right)_{3}$-Catalyzed Hydrosilylation of Vinylcyclopropanes. Org. Lett. 2020, 22, 73837386.

(12) Chandu, P.; Ghosh, K. G.; Sureshkumar, D., Metal-Free Visible-Light-Promoted Trifluoromethylation of Vinylcyclopropanes Using Pyrylium Salt as a Photoredox Catalyst. J. Org. Chem. 2019, $84,8771-8781$

(13) (a) Liang, Z.; Wang, F.; Chen, P.; Liu, G., Coppercatalyzed intermolecular cyanotrifluoromethylation of alkenes: Convenient synthesis of $\mathrm{CF}_{3}$-containing alkyl nitriles. J. Fluorine Chem. 2014, 167, 55-60. (b) Fu, L.; Zhou, S.; Wan, X.; Chen, P.; Liu, G., Enantioselective Trifluoromethylalkynylation of Alkenes via CopperCatalyzed Radical Relay. J. Am. Chem. Soc. 2018, 140, 10965-1096.

$\begin{array}{lll}\text { (14) Dunkelblum, E., Addition reactions to } & \text { ren } \\ \text { ethylenecyclopropanes-III: } & \text { The reaction of 2,4- }\end{array}$ dinitrobenzenesulfenyl chloride with alkylidenecyclopropanes. Tetrahedron 1974, 30, 3991-3996.

(15) Liu, J.; Yao, H.; Li, X.; Wu, H.; Lin, A.; Yao, H.; Xu, J.; $\mathrm{Xu}, \quad$ S., Organocatalytic 1,5-trifluoromethylthio-sulfonylation of vinylcyclopropane mediated by visible light in the water phase. Org. Chem. Front. 2020, 7, 1314-1320.
(16) (a) Luo, Q.; Mao, R.; Zhu, Y.; Wang, Y., PhotoredoxCatalyzed Generation of Sulfamyl Radicals: Sulfonamidation of Enol Silyl Ether with Chlorosulfonamide. J. Org. Chem. 2019, 84, 1389713907. (b) Griller, D.; Ingold, K. U., Free-radical clocks. Acc. Chem. Res. 1980, 13, 317-323. (c) Li, J.-M.; Wang, Y.-H.; Yu, Y.; Wu, R.B.; Weng, J.; Lu, G., Copper-Catalyzed Remote C-H Functionalizations of Naphthylamides through a Coordinating Activation Strategy and Single-Electron-Transfer (SET) Mechanism. ACS Catalysis 2017, 7, 2661-2667. (d) Nie, X.; Xu, T.; Song, J.; Devaraj, A.; Zhang, B.; Chen, Y.; Liao, S., Radical Fluorosulfonylation: Accessing Alkenyl Sulfonyl Fluorides from Alkenes. Angew. Chem. Int. Ed. 2021, 60, 3956-3960. (e) Yuan, Y.; Cao, Y.; Lin, Y.; Li, Y.; Huang, Z.; Lei, A., Electrochemical Oxidative Alkoxysulfonylation of Alkenes Using Sulfonyl Hydrazines and Alcohols with Hydrogen Evolution. ACS Catalysis 2018, 8, 10871-10875. (f) Kristensen, S. K.; Laursen, S. L. R.; Taarning, E.; Skrydstrup, T., Ex-Situ Formation of Methanethiol: Application in the Gold(I)-Promoted AntiMarkovnikov Hydrothiolation of Olefins. Angew. Chem. Int. Ed. 2018, 57, 13887-13891. (g) Zheng, Y.; You, Y.; Shen, Q.; Zhang, J.; Liu, L.; Duan, X.-H., Visible-light-induced anti-Markovnikov hydrosulfonation of styrene derivatives. Org. Chem. Front. 2020, 7, 2069-2074.

(17) (a) Sowaileh, M. F.; Hazlitt, R. A.; Colby, D. A., Application of the Pentafluorosulfanyl Group as a Bioisosteric Replacement. ChemMedChem 2017, 12, 1481-1490. (b) Savoie, P. R.; Welch, J. T., Preparation and utility of organic pentafluorosulfanyl-containing compounds. Chem. Rev. 2015, 115, 1130-1190.

(18) (a) Beier, P., Pentafluorosulfanylation of Aromatics and Heteroaromatics. In Emerging Fluorinated Motifs, Ma, J.-A.; Cahard, D., Eds. Wiley-VCH: Weinheim, Germany, 2020; Vol. 2, pp 551570. (b) Debrauwer, V.; Leito, I.; Lõkov, M.; Tshepelevitsh, S.; Parmentier, M.; Blanchard, N.; Bizet, V., Synthesis and Physicochemical Properties of 2-SF5-(Aza)Indoles, a New Family of $\mathrm{SF}_{5}$ Heterocycles. ACS Org. Inorg. Au 2021, DOI 10.1021/acsorginorgau.1c00010.

(19) (a) Haufe, G., Pentafluorosulfanylation of Aliphatic Substrates. In Emerging Fluorinated Motifs, Ma, J.-A.; Cahard, D., Eds.: Weinheim, Germany, 2020; Vol. 2, pp 571-609. (b) Lefebvre, G.; Charron, O.; Cossy, J.; Meyer, C., Radical Addition of $\mathrm{SF}_{5} \mathrm{Cl}$ to Cyclopropenes: Synthesis of (Pentafluorosulfanyl)cyclopropanes. Org. Lett. 2021, 23, 5491-5495.

(20) (a) Pitts, C. R.; Santschi, N.; Togni, A. Method for preparing polyfluorinated compound. WO2019229103A1, 2019. (b) Shou, J. Y.; Xu, X. H.; Qing, F. L., Chemoselective Hydro(Chloro)pentafluorosulfanylation of Diazo Compounds with Pentafluorosulfanyl Chloride. Angew. Chem. Int. Ed. 2021, 60, 15271-15275. (c) Burton, D. J.; Wang, Y.; Bizet, V.; Cahard, D., Pentafluorosulfanyl Chloride. In Encyclopedia of Reagents for Organic Synthesis, DOI org/10.1002/047084289X.rn00413.pub2.

(21) (a) Mohand, S. A.; Dolbier, W. R., Jr., New and Convenient Method for Incorporation of Pentafluorosulfanyl $\left(\mathrm{SF}_{5}\right)$ Substituents into Aliphatic Organic Compounds. Org. Lett. 2002, 4, 30133015. (b) Gilbert, A.; Paquin, J.-F., Evaluation of the compatibility of pentafluorosulfanyl chloride with various solvents and additives. $J$. Fluorine Chem. 2019, 221, 70-74. (c) Gilbert, A.; Langowski, P.; Delgado, M.; Chabaud, L.; Pucheault, M.; Paquin, J.-F., Amineborane complex-initiated $\mathrm{SF}_{5} \mathrm{Cl}$ radical addition on alkenes and alkynes. Beilstein J. Org. Chem. 2020, 16, 3069-3077. (d) Das, P.; Takada, M.; Tokunaga, E.; Saito, N.; Shibata, N., Synthesis of pyridine trans-tetrafluoro- $\lambda^{6}$-sulfane derivatives via radical addition. Org. Chem. Front. 2018, 5, 719-724. (e) Niina, K.; Tanagawa, K.; Sumii, Y.; Saito, N.; Shibata, N., Pyridine tetrafluoro- $\lambda^{6}$-sulfanyl chlorides: spontaneous addition to alkynes and alkenes in the presence or absence of photo-irradiation. Org. Chem. Front. 2020, 7, 1276-1282.

(22) CCDC 2088835 (3o) contains the crystallographic data for this paper. These data can be obtained free of charge from The Cambridge Crystallographic Data Centre via www. ccdc.cam.ac.uk/data_request/cif.

(23) Campredon, M.; Kanabus-Kaminska, J. M.; Griller, D., Conformational control of regioselectivity: rearrangements of 
cyclopropylcarbinyl and 5-substituted pent-3-enyl radicals. J. Org. Chem. 1988, 53, 5393-5396.

(24) (a) Kleemann, G.; Seppelt, K., Methylsulfurpentafluoride and some of its derivatives. Chem. Ber. 1979, 112, 1140-1146. (b) Henkel, T.; Krügerke, T.; Seppelt, K., Isomerisierung von Benzoylalkylidenschwefeltetrafluoriden $\quad \mathrm{C}_{6} \mathrm{H}_{5}-\mathrm{COCR}=\mathrm{SF}_{4} \quad \mathrm{zu}$ Dihydrooxathiet-Derivaten. Angew. Chem. 1990, 102, 1171-1172. (c) Dolbier, W. R.; Ait-Mohand, S.; Schertz, T. D.; Sergeeva, T. A.; Cradlebaugh, J. A.; Mitani, A.; Gard, G. L.; Winter, R. W.; Thrasher, J. S., A convenient and efficient method for incorporation of pentafluorosulfanyl $\left(\mathrm{SF}_{5}\right)$ substituents into aliphatic compounds. $J$. Fluorine Chem. 2006, 127, 1302-1310. (d) Welch, J.; Ngo, S.; Lim, D. Alpha-pentafluorosufanyl aldehydes, ketones and acids. WO2009026191A1, 2009. (e) Cloutier, M.; Roudias, M.; Paquin, J.F., Regioselective Gold-Catalyzed Hydration of $\mathrm{CF}_{3^{-}}$and $\mathrm{SF}_{5^{-}}$ alkynes. Org. Lett. 2019, 21, 3866-3870. (f) Shou, J. Y.; Xu, X. H.; Qing, F. L., Chemoselective Hydro(Chloro)pentafluorosulfanylation of Diazo Compounds with Pentafluorosulfanyl Chloride. Angew. Chem. Int. Ed. 2021, 60, 15271-15275.
(25) (a) Wang, X.; Wang, X.; Liu, M.; Ding, J.; Chen, J.; Wu, H., Palladium-Catalyzed Reaction of Arylboronic Acids with Aliphatic Nitriles: Synthesis of Alkyl Aryl Ketones and 2-Arylbenzofurans. Synthesis 2013, 2241-2244. (b) Liu, J.; Yao, H.; Li, X.; Wu, H.; Lin, A.; Yao, H.; Xu, J.; Xu, S., Organocatalytic 1,5-trifluoromethylthiosulfonylation of vinylcyclopropane mediated by visible light in the water phase. Org. Chem. Front. 2020, 7, 1314-1320.

(26) Long, P.-W.; He, T.; Oestreich, M., B $\left(\mathrm{C}_{6} \mathrm{~F}_{5}\right)_{3}$-Catalyzed Hydrosilylation of Vinylcyclopropanes. Org. Lett. 2020, 22, 73837386.

(27) (a) Ciaccio, J. A.; Aman, C. E., "Instant Methylide" Modification of the Corey-Chaykovsky Cyclopropanation Reaction. Synth. Commun. 2006, 36, 1333-1341. (b) Zhang, Z.-Q.; Meng, X.-Y.; Sheng, J.; Lan, Q.; Wang, X.-S., Enantioselective Copper-Catalyzed 1,5-Cyanotrifluoromethylation of Vinylcyclopropanes. Org. Lett. 2019, 21, 8256-8260.

(28) Kong, J.-R.; Ngai, M.-Y.; Krische, M. J., Highly Enantioselective Direct Reductive Coupling of Conjugated Alkynes and $\alpha$-Ketoesters via Rhodium-Catalyzed Asymmetric Hydrogenation. J. Am. Chem. Soc. 2006, 128, 718-719 